\title{
Optimization design of structures subjected to transient loads using first and second derivatives of dynamic displacement and
} stress

\author{
Qimao Liu ${ }^{\mathrm{a}, *}$, Jing Zhang ${ }^{\mathrm{c}}$ and Liubin Yan ${ }^{\mathrm{b}}$ \\ ${ }^{a}$ Department of Civil and Structural Engineering, Aalto University, Espoo, Finland \\ ${ }^{\mathrm{b}}$ College of Civil and Architecture Engineering, Guangxi University, Nanning, China \\ ${ }^{\mathrm{c}}$ Department of Mechanical Engineering, Indiana University-Purdue University Indianapolis, Indianapolis, IN, \\ USA
}

Received 16 September 2010

Revised 19 July 2011

\begin{abstract}
This paper developed an effective optimization method, i.e., gradient-Hessian matrix-based method or second order method, of frame structures subjected to the transient loads. An algorithm of first and second derivatives of dynamic displacement and stress with respect to design variables is formulated based on the Newmark method. The inequality time-dependent constraint problem is converted into a sequence of appropriately formed time-independent unconstrained problems using the integral interior point penalty function method. The gradient and Hessian matrixes of the integral interior point penalty functions are also computed. Then the Marquardt's method is employed to solve unconstrained problems. The numerical results show that the optimal design method proposed in this paper can obtain the local optimum design of frame structures and sometimes is more efficient than the augmented Lagrange multiplier method.
\end{abstract}

Keywords: Dynamic response optimization, gradient, Hessian matrix, time-dependent constraint

\section{Introduction}

Dynamic response analysis of structures subjected to transient loads often requires much more demanding computational cost than static analysis. Therefore the efficiency of the optimization method becomes critical to dynamic response optimization problems. Mathematically, there are three types of dynamic response optimization methods: zero order methods (nongradient-based algorithms), first order methods (gradient-based algorithms) and second order methods (gradient-Hessian matrix-based algorithms). Generally, the first order methods are more efficient than the zero order methods, and the second order methods are more efficient than the first order methods. Zero order methods require only the information of dynamic responses to construct optimal algorithms. A lot of nongradient-based algorithms [1-4] have been developed to solve the optimization problem of structures subjected to transient loads. First order methods require the information of dynamic responses and their first derivatives with respect to design variables to construct optimal algorithms. Therefore, it is very crucial to calculate efficiently the

\footnotetext{
* Corresponding author: Qimao Liu, Department of Civil and Structural Engineering, Aalto University, Espoo, FI-00076 Aalto, Finland. Tel.: +3589470 23701; Fax: +3589470 23758; E-mail: liuqimao2005@163.com.
} 
first derivatives of structural dynamic responses for gradient-based algorithms. However, many algorithms have been developed to calculate dynamic response first derivatives with respect to design variables (also called sensitivity analysis [5-12]). Today, the gradient-based algorithms are the mainstream pattern [13] to solve the optimization problem of structures subjected to transient loads. Much research [14-17] has been done to solve the structural dynamic response optimization problem with the gradient-based algorithms. Second order methods require the information of dynamic responses, their first and second derivatives with respect to design variables to construct optimal algorithms. The Newton's method and Quasi Newton's method are the commonly used second order method. Although many researchers have calculated dynamic response first derivatives with respect to design variables, there is little literature published on dynamic response second derivative analysis (also called Hessian matrix analysis). Second derivative analysis is more complicated than first derivative analysis. However, the efficiency of the optimization using second derivative can be greatly improved when the gradient and Hessian matrix can be calculated efficiently. In this paper, a gradient-Hessian matrix-based algorithm for optimization problem of structures subjected to transient loads is developed based on gradient and Hessian matrix calculations.

The purpose of this paper is to develop a gradient-Hessian matrix-based optimization method for structures subjected to dynamic loads. The main work of this paper is as follows: First, we formulate an algorithm to calculate dynamic responses and their first and second derivatives with respect to design variables. The algorithm is achieved by direct differentiation and only a single dynamics analysis, based on Newmark- $\beta$ method [18], is required. Second, we formulate the time-dependent structural optimization model. In this model, total mass of the structure is the objective function. The dynamic responses including stresses of the beam element and nodal displacements are constraints. Third, the time-dependent optimization model is converted into a sequence of appropriately formed unconstrained integral mathematic models using the interior penalty function method. The gradient and Hessian matrixes of the interior penalty functions are also calculated using the first and second derivatives of dynamic responses. Fourth, Marquardt's method [19], a gradient-Hessian matrix-based algorithm, is employed to solve the unconstrained integral mathematic model. Finally, as the illustration of the developed approach, optimization designs of a plane frame subjected to the horizontal dynamic loads are demonstrated.

\section{Calculation of first and second derivatives}

This section presents two subjects. Section 2.1 introduces the calculations of first and second derivatives of dynamic responses (i.e., nodal displacement and normal stress) with respect to design variables of structures. Section 2.2 discusses how to calculate first and second derivatives of structural mass with respect to structural design variables.

\subsection{Calculation of first and second derivatives of dynamic displacement and stress}

The first and second derivatives of dynamic response of structures are the prerequisites of an efficient gradientHessian matrix-based algorithm. We will show here that, in a single structural dynamic analysis, the first and second derivatives of a dynamic response can be derived simultaneously.

Plane beam element is used extensively in engineering. Therefore, the dynamic optimal design method is demonstrated with the plane beam element in this work. The element cross-section shown in Fig. 1 is $\mathrm{H}$ structural-steel shape. The design variables of element $e$ are $d_{1}^{e}, d_{2}^{e}, d_{3}^{e}$ and $d_{4}^{e}$. Point $c$ is the centroid of the element cross-section. Axes $y$ and $z$ are the principal centroidal axes.

The cross-sectional area is

$$
A^{e}=2 d_{1}^{e} d_{3}^{e}+d_{2}^{e} d_{4}^{e}
$$

The cross-sectional moment of inertia is

$$
I_{z}^{e}=\frac{d_{4}^{e} d_{2}^{e^{3}}}{12}+2\left[\frac{d_{1}^{e} d_{3}^{e 3}}{12}+\left(\frac{d_{3}^{e}+d_{2^{e}}}{2}\right)^{2} d_{1}^{e} d_{3}^{e}\right]
$$

The farthest distance $y$ from the neutral axis is 


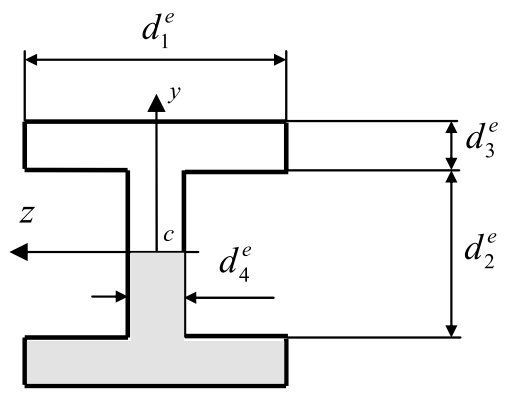

Fig. 1. Element cross-section and element design variables.

$$
y_{m}^{e}= \pm\left(d_{3}^{e}+\frac{d_{2}^{e}}{2}\right)
$$

The first moment of the shaded area with respect to the neutral axis is

$$
S_{z \max }^{e *}=d_{1}^{e} d_{3}^{e} \frac{d_{3}^{e}+d_{2}^{e}}{2}+\frac{d_{2}^{e}}{2} d_{4}^{e} \frac{d_{2}^{e}}{4}
$$

The structure is divided with $N$ elements. The design variable vector is defined as.

$$
\mathbf{d}=\left[d_{1}^{1}, d_{2}^{1}, d_{3}^{1}, d_{4}^{1}, \cdots, d_{1}^{e}, d_{2}^{e}, d_{3}^{e}, d_{4}^{e}, \cdots, d_{1}^{N}, d_{2}^{N}, d_{3}^{N}, d_{4}^{N}\right]^{T}
$$

Consider the equations of motion for a linear system subjected to dynamic forces

$$
\mathbf{M} \ddot{\mathbf{x}}+\mathbf{C} \dot{\mathbf{x}}+\mathbf{K} \mathbf{x}=\mathbf{f}(t)
$$

with the following initial conditions:

$$
\left\{\begin{array}{l}
\mathbf{x}(0)=\mathbf{x}_{0} \\
\dot{\mathbf{x}}(0)=\dot{\mathbf{x}}_{0}
\end{array}\right.
$$

where $\mathbf{K}, \mathbf{M}$, and $\mathbf{C}$ are stiffness matrix, mass matrix , and damping matrix, respectively. $\mathbf{x}(t), \dot{\mathbf{x}}(t)$ and $\ddot{\mathbf{x}}(t)$ are unknown nodal displacement, velocity and acceleration vectors, and $\mathbf{f}(t)$ is the load vector. Suppose that the dynamic loads act on the nodes.

Rayleigh damping is used in this work, the structural damping matrix is

$$
\mathbf{C}=\alpha_{1} \mathbf{M}+\alpha_{2} \mathbf{K}
$$

where

$$
\begin{aligned}
\alpha_{1} & =\frac{2\left(\frac{\varsigma_{1}}{\omega_{1}}-\frac{\varsigma_{2}}{\omega_{2}}\right)}{\frac{1}{\omega_{1}^{2}}-\frac{1}{\omega_{2}^{2}}} \\
\alpha_{2} & =\frac{2\left(\varsigma_{2} \omega_{2}-\varsigma_{1} \omega_{1}\right)}{\omega_{2}^{2}-\omega_{1}^{2}}
\end{aligned}
$$

where $\omega_{1}$ and $\omega_{2}$ are the first and second natural frequency of the structure, respectively. $\varsigma_{1}$ and $\varsigma_{2}$ are the damping ratio. In this work, $\varsigma_{1}=\varsigma_{2}=0.02 . \alpha_{1}$ and $\alpha_{2}$ are constants.

Equations (6) and (7) must be satisfied for all time period $t \in[0, \Gamma]$. $\Gamma$ is the duration of the dynamic loads. In practice, the solution of this initial-boundary-value problem (IBVP) requires integration through time. This is achieved numerically by discretising in time the continuous temporal derivatives that appear in the equation. Any one of the time integration procedures can be used for this purpose. The most widely used family of direct time integration methods for solving Eq. (6) is the Newmark family of methods. The Newmark method can be formulated by considering equilibrium at any discrete time $t+\Delta t$, and is given by the following equation:

$$
\mathbf{M} \ddot{\mathbf{x}}(t+\Delta t)+\mathbf{C} \dot{\mathbf{x}}(t+\Delta t)+\mathbf{K} \mathbf{x}(t+\Delta t)=\mathbf{f}(t+\Delta t)
$$


The nodal displacements, nodal accelerations and nodal velocities can be achieved at any time $t+\Delta t$ by solving Eq. (11).

Perhaps the most widely used direct method for the equation of motion (6) is the Newmark method, an implicit technique, which consists of the following finite difference assumptions with regard to the evolution of the approximate solution:

$$
\begin{aligned}
& \mathbf{x}(t+\Delta t)=\mathbf{x}(t)+\Delta t \dot{\mathbf{x}}(t)+\Delta t^{2}\left[\left(\frac{1}{2}-\beta\right) \ddot{\mathbf{x}}(t)+\beta \ddot{\mathbf{x}}(t+\Delta t)\right] \\
& \dot{\mathbf{x}}(t+\Delta t)=\dot{\mathbf{x}}(t)+\Delta t[(1-\delta) \ddot{\mathbf{x}}(t)+\delta \ddot{\mathbf{x}}(t+\Delta t)]
\end{aligned}
$$

where any particular choice of the parameters $\beta$ and $\delta$ determines the stability and accuracy characteristics of the solution. In this work, parameters $\delta \geqslant 0.5$ and $\beta=0.25(0.5+\delta)^{2}$. We define the integral constants: $a_{0}=\frac{1}{\beta \Delta t^{2}}$, $a_{1}=\frac{\delta}{\beta \Delta t}, a_{2}=\frac{1}{\beta \Delta t}, a_{3}=\frac{1}{2 \beta}-1, a_{4}=\frac{\delta}{\beta}-1, a_{5}=\frac{\Delta t}{2}\left(\frac{\delta}{\beta}-2\right), a_{6}=\Delta t(1-\delta), a_{7}=\delta \Delta t$. The parameters $\beta$ and $\delta$ will be replaced by those constants in the following formulas.

In addition to Eqs (12) and (13) the equilibrium Eq. (11) at time station $t+\Delta t$ is considered. This way a system of equations is formed for the determination of the three unknowns $\mathbf{x}(t+\Delta t), \dot{\mathbf{x}}(t+\Delta t)$ and $\ddot{\mathbf{x}}(t+\Delta t)$, assuming that the displacement, velocity, and acceleration vectors at the previous time station $t$ have already been computed. Thus, the solution for the displacement vector is

$$
\mathbf{K}^{*} \mathbf{x}(t+\Delta t)=\mathbf{F}^{*}(t+\Delta t)
$$

where

$$
\mathbf{K}^{*}=\mathbf{K}+a_{0} \mathbf{M}+a_{1} \mathbf{C}
$$

and

$$
\mathbf{F}^{*}(t+\Delta t)=\mathbf{f}(t+\Delta t)+\mathbf{M}\left[a_{0} \mathbf{x}(t)+a_{2} \dot{\mathbf{x}}(t)+a_{3} \ddot{\mathbf{x}}(t)\right]+\mathbf{C}\left[a_{1} \mathbf{x}(t)+a_{4} \dot{\mathbf{x}}(t)+a_{5} \ddot{\mathbf{x}}(t)\right]
$$

The accelerations, $\ddot{\mathbf{x}}(t+\Delta t)$, which are required for the computations at the next time station, can be computed as

$$
\ddot{\mathbf{x}}(t+\Delta t)=a_{0}[\mathbf{x}(t+\Delta t)-\mathbf{x}(t)]-a_{2} \dot{\mathbf{x}}(t)-a_{3} \ddot{\mathbf{x}}(t)
$$

while the velocities, $\dot{\mathbf{x}}(t+\Delta t)$, can be obtained directly from equation (13), as follows.

$$
\dot{\mathbf{x}}(t+\Delta t)=\dot{\mathbf{x}}(t)+a_{6} \ddot{\mathbf{x}}(t)+a_{7} \ddot{\mathbf{x}}(t+\Delta t)
$$

When the dynamic loads act on the nodes, we only need to determine the internal forces and internal couples at the center and two ends of the element. The element nodal force vector, $\overline{\mathbf{F}}^{e}(t+\Delta t)$, is

$$
\overline{\mathbf{F}}^{e}(t+\Delta t)=\overline{\mathbf{K}}^{e} \mathbf{T}^{e} \delta^{e}(t+\Delta t)
$$

where $\overline{\mathbf{K}}^{e}$ is the element stiffness matrix in a local coordinate system, $\mathbf{T}^{e}$ is the element coordinate transformation matrix, $\delta^{e}(t+\Delta t)$ is the element nodal displacement vector in a local coordinate system.

The internal force vector at the center of element, $\overline{\mathbf{F}}_{M}^{e}(t+\Delta t)$, is

$$
\overline{\mathbf{F}}_{M}^{e}(t+\Delta t)=\left[\begin{array}{lll}
-\overline{\mathbf{F}}_{1}^{e}(t+\Delta t) & -\overline{\mathbf{F}}_{2}^{e}(t+\Delta t) & \frac{l_{e}}{2} \overline{\mathbf{F}}_{2}^{e}(t+\Delta t)-\overline{\mathbf{F}}_{3}^{e}(t+\Delta t)
\end{array}\right]^{T}
$$

where $l_{e}$ is the length of element. The three terms, i.e., $-\overline{\mathbf{F}}_{1}^{e}(t+\Delta t),-\overline{\mathbf{F}}_{2}^{e}(t+\Delta t)$ and $\frac{l_{e}}{2} \overline{\mathbf{F}}_{2}^{e}(t+\Delta t)-$ $\overline{\mathbf{F}}_{3}^{e}(t+\Delta t)$, are axial force, shear force and bending moment, respectively. $\overline{\mathbf{F}}_{1}^{e}(t+\Delta t), \mathbf{F}_{2}^{e}(t+\Delta t)$ and $\overline{\mathbf{F}}_{3}^{e}(t+\Delta t)$ are the first, second and third term of the element nodal force vector, $\overline{\mathbf{F}}^{e}(t+\Delta t)$, respectively.

The maximum stress at the center and two ends, i.e. the $i$ end and the $j$ end, of the element are calculated as follows.

The maximum normal stress at the $i$ end of the element is

$$
\sigma_{i x}^{e}(t+\Delta t)=\frac{\overline{\mathbf{F}}_{1}^{e}(t+\Delta t)}{A^{e}}+\frac{\overline{\mathbf{F}}_{3}^{e}(t+\Delta t) y_{m}^{e}}{E I_{z}^{e}}
$$


The maximum normal stress at the $j$ end of the element is

$$
\sigma_{j x}^{e}(t+\Delta t)=\frac{\overline{\mathbf{F}}_{4}^{e}(t+\Delta t)}{A^{e}}+\frac{\overline{\mathbf{F}}_{6}^{e}(t+\Delta t) y_{m}^{e}}{E I_{z}^{e}}
$$

where $\overline{\mathbf{F}}_{4}^{e}(t+\Delta t)$ and $\overline{\mathbf{F}}_{6}^{e}(t+\Delta t)$ are the fourth and sixth term of the element nodal force vector, $\overline{\mathbf{F}}^{e}(t+\Delta t)$, respectively.

The maximum normal stress at the center of the element is

$$
\sigma_{M x}^{e}(t+\Delta t)=\frac{\overline{\mathbf{F}}_{M 1}^{e}(t+\Delta t)}{A^{e}}+\frac{\overline{\mathbf{F}}_{M 3}^{e}(t+\Delta t) y_{m}^{e}}{E I_{z}^{e}}
$$

where $\overline{\mathbf{F}}_{\underline{M} 1}^{e}(t+\Delta t)$ and $\overline{\mathbf{F}}_{M 3}^{e}(t+\Delta t)$ are the first and third term of the internal force vector at the center of element, $\overline{\mathbf{F}}_{M}^{e}(t+\Delta t)$, respectively.

The maximum shear stress at the $i$ end of the element is

$$
\tau_{i x y}^{e}(t+\Delta t)=\frac{\overline{\mathbf{F}}_{2}^{e}(t+\Delta t) S_{z \max }^{e *}}{d_{4}^{e} I_{z}^{e}}
$$

The maximum shear stress at the $j$ end of the element is

$$
\tau_{j x y}^{e}(t+\Delta t)=\frac{\overline{\mathbf{F}}_{5}^{e}(t+\Delta t) S_{z \max }^{e *}}{d_{4}^{e} I_{z}^{e}}
$$

where $\overline{\mathbf{F}}_{5}^{e}(t+\Delta t)$ is the fifth term of the element nodal force vector, $\overline{\mathbf{F}}^{e}(t+\Delta t)$.

The maximum shear stress at the center of the element is

$$
\tau_{M x y}^{e}(t+\Delta t)=\frac{\overline{\mathbf{F}}_{M 2}^{e}(t+\Delta t) S_{z \max }^{e *}}{d_{4}^{e} I_{z}^{e}}
$$

where $\overline{\mathbf{F}}_{M 2}^{e}(t+\Delta t)$ is the second term of the internal force vector at the center of element, $\overline{\mathbf{F}}_{M}^{e}(t+\Delta t)$.

\subsubsection{Formulas for first derivatives of dynamic displacement and stress}

Now we will derive the formulas for the first derivatives of dynamic response, i.e., dynamic displacement and stress. Differentiating Eq. (14) with respect to the design variable $d_{i}$, we have

$$
\mathbf{K}^{*} \frac{\partial \mathbf{x}(t+\Delta t)}{\partial d_{i}}=\frac{\partial \mathbf{F}^{*}(t+\Delta t)}{\partial d_{i}}-\frac{\partial \mathbf{K}^{*}}{\partial d_{i}} \mathbf{x}(t+\Delta t)
$$

where

$$
\frac{\partial \mathbf{K}^{*}}{\partial d_{i}}=\frac{\partial \mathbf{K}}{\partial d_{i}}+a_{0} \frac{\partial \mathbf{M}}{\partial d_{i}}+a_{1} \frac{\partial \mathbf{C}}{\partial d_{i}}
$$

and

$$
\begin{aligned}
& \frac{\partial \mathbf{F}^{*}(t+\Delta t)}{\partial d_{i}}=\frac{\partial \mathbf{M}}{\partial d_{i}}\left[a_{0} \mathbf{x}(t)+a_{2} \dot{\mathbf{x}}(t)+a_{3} \ddot{\mathbf{x}}(t)\right]+\mathbf{M}\left[a_{0} \frac{\partial \mathbf{x}(t)}{\partial d_{i}}+a_{2} \frac{\partial \dot{\mathbf{x}}(t)}{\partial d_{i}}+a_{3} \frac{\partial \ddot{\mathbf{x}}(t)}{\partial d_{i}}\right] \\
& +\frac{\partial \mathbf{C}}{\partial d_{i}}\left[a_{1} \mathbf{x}(t)+a_{4} \dot{\mathbf{x}}(t)+a_{5} \ddot{\mathbf{x}}(t)\right]+\mathbf{C}\left[a_{1} \frac{\partial \mathbf{x}(t)}{\partial d_{i}}+a_{4} \frac{\partial \dot{\mathbf{x}}(t)}{\partial d_{i}}+a_{5} \frac{\partial \ddot{\mathbf{x}}(t)}{\partial d_{i}}\right]
\end{aligned}
$$

After the first derivatives of displacement vector at time $t+\Delta t$ is obtained from Eq. (27), through differentiating Eq. (17) with respect to design variables $d_{i}$, we have

$$
\frac{\partial \ddot{\mathbf{x}}(t+\Delta t)}{\partial d_{i}}=a_{0}\left[\frac{\partial \mathbf{x}(t+\Delta t)}{\partial d_{i}}-\frac{\partial \mathbf{x}(t)}{\partial d_{i}}\right]-a_{2} \frac{\partial \dot{\mathbf{x}}(t)}{\partial d_{i}}-a_{3} \frac{\partial \ddot{\mathbf{x}}(t)}{\partial d_{i}}
$$

Then, the first derivatives of acceleration vector at time $t+\Delta t$ is obtained from Eq. (30). Differentiating Eq. (18) with respect to design variable $d_{i}$, we have

$$
\frac{\partial \dot{\mathbf{x}}(t+\Delta t)}{\partial d_{i}}=\frac{\partial \dot{\mathbf{x}}(t)}{\partial d_{i}}+a_{6} \frac{\partial \ddot{\mathbf{x}}(t)}{\partial d_{i}}+a_{7} \frac{\partial \ddot{\mathbf{x}}(t+\Delta t)}{\partial d_{i}}
$$


Differentiating Eq. (19) with respect to the design variable $d_{i}$, we have

$$
\frac{\partial \overline{\mathbf{F}}^{e}(t+\Delta t)}{\partial d_{i}}=\frac{\partial \overline{\mathbf{K}}^{e}}{\partial d_{i}} \mathbf{T}^{e} \delta^{e}(t+\Delta t)+\overline{\mathbf{K}}^{e} \mathbf{T}^{e} \frac{\partial \delta^{e}(t+\Delta t)}{\partial d_{i}}
$$

where $\frac{\partial \delta^{e}(t+\Delta t)}{\partial d_{i}}$ can be calculated from $\frac{\partial \mathbf{x}(t+\Delta t)}{\partial d_{i}}$.

Differentiating Eq. (20) with respect to the design variable $d_{i}$, we have

$$
\frac{\partial \overline{\mathbf{F}}_{M}^{e}(t+\Delta t)}{\partial d_{i}}=\left[\begin{array}{lll}
-\frac{\partial \overline{\mathbf{F}}_{1}^{e}(t+\Delta t)}{\partial d_{i}} & -\frac{\partial \overline{\mathbf{F}}_{2}^{e}(t+\Delta t)}{\partial d_{i}} & \frac{l_{e}}{2} \frac{\partial \overline{\mathbf{F}}_{2}^{e}(t+\Delta t)}{\partial d_{i}}-\frac{\partial \overline{\mathbf{F}}_{3}^{e}(t+\Delta t)}{\partial d_{i}}
\end{array}\right]^{T}
$$

Differentiating Eq. (21) with respect to the design variable $d_{i}$, we have

$$
\begin{aligned}
& \frac{\partial \sigma_{i x}^{e}(t+\Delta t)}{\partial d_{i}}=\frac{\partial \overline{\mathbf{F}}_{1}^{e}(t+\Delta t)}{\partial d_{i}} \frac{1}{A^{e}}-\frac{\overline{\mathbf{F}}_{1}^{e}(t+\Delta t)}{A^{e 2}} \frac{\partial A^{e}}{\partial d_{i}} \\
& +\frac{\partial \overline{\mathbf{F}}_{3}^{e}(t+\Delta t)}{\partial d_{i}} \frac{y_{m}^{e}}{E I_{z}^{e}}+\frac{\overline{\mathbf{F}}_{3}^{e}(t+\Delta t)}{E I_{z}^{e}} \frac{\partial y_{m}^{e}}{\partial d_{i}}-\frac{\overline{\mathbf{F}}_{3}^{e}(t+\Delta t) y_{m}^{e}}{E I_{z}^{e 2}} \frac{\partial I_{z}^{e}}{\partial d_{i}}
\end{aligned}
$$

Differentiating Eq. (22) with respect to the design variable $d_{i}$, we have

$$
\begin{aligned}
& \frac{\partial \sigma_{j x}^{e}(t+\Delta t)}{\partial d_{i}}=\frac{\partial \overline{\mathbf{F}}_{4}^{e}(t+\Delta t)}{\partial d_{i}} \frac{1}{A^{e}}-\frac{\overline{\mathbf{F}}_{4}^{e}(t+\Delta t)}{A^{e 2}} \frac{\partial A^{e}}{\partial d_{i}} \\
& +\frac{\partial \overline{\mathbf{F}}_{6}^{e}(t+\Delta t)}{\partial d_{i}} \frac{y_{m}^{e}}{E I_{z}^{e}}+\frac{\overline{\mathbf{F}}_{6}^{e}(t+\Delta t)}{E I_{z}^{e}} \frac{\partial y_{m}^{e}}{\partial d_{i}}-\frac{\overline{\mathbf{F}}_{6}^{e}(t+\Delta t) y_{m}^{e}}{E I_{z}^{e 2}} \frac{\partial I_{z}^{e}}{\partial d_{i}}
\end{aligned}
$$

Differentiating Eq. (23) with respect to the design variable $d_{i}$, we have

$$
\begin{aligned}
& \frac{\partial \sigma_{M x}^{e}(t+\Delta t)}{\partial d_{i}}=\frac{\partial \overline{\mathbf{F}}_{M 1}^{e}(t+\Delta t)}{\partial d_{i}} \frac{1}{A^{e}}-\frac{\overline{\mathbf{F}}_{M 1}^{e}(t+\Delta t)}{A^{e 2}} \frac{\partial A^{e}}{\partial d_{i}} \\
& +\frac{\partial \overline{\mathbf{F}}_{M 3}^{e}(t+\Delta t)}{\partial d_{i}} \frac{y_{m}^{e}}{E I_{z}^{e}}+\frac{\overline{\mathbf{F}}_{M 3}^{e}(t+\Delta t)}{E I_{z}^{e}} \frac{\partial y_{m}^{e}}{\partial d_{i}}-\frac{\overline{\mathbf{F}}_{M 3}^{e}(t+\Delta t) y_{m}^{e}}{E I_{z}^{e 2}} \frac{\partial I_{z}^{e}}{\partial d_{i}}
\end{aligned}
$$

Differentiating Eq. (24) with respect to the design variable $d_{i}$, we have

$$
\begin{aligned}
& \frac{\partial \tau_{i x y}^{e}(t+\Delta t)}{\partial d_{i}}=\frac{\partial \overline{\mathbf{F}}_{2}^{e}(t+\Delta t)}{\partial d_{i}} \frac{S_{z \max }^{e *}}{d_{4}^{e} I_{z}^{e}}+\frac{\partial S_{z \max }^{e *}}{\partial d_{i}} \frac{\overline{\mathbf{F}}_{2}^{e}(t+\Delta t)}{d_{4}^{e} I_{z}^{e}} \\
& -\frac{\partial d_{4}^{e}}{\partial d_{i}} \frac{\overline{\mathbf{F}}_{2}^{e}(t+\Delta t) S_{z \max }^{e *}}{d_{4}^{e 2} I_{z}^{e}}-\frac{\partial I_{z}^{e}}{\partial d_{i}} \frac{\overline{\mathbf{F}}_{2}^{e}(t+\Delta t) S_{z \max }^{e *}}{d_{4}^{e} I_{z}^{e 2}}
\end{aligned}
$$

Differentiating Eq. (25) with respect to the design variable $d_{i}$, we have

$$
\begin{aligned}
& \frac{\partial \tau_{j x y}^{e}(t+\Delta t)}{\partial d_{i}}=\frac{\partial \overline{\mathbf{F}}_{5}^{e}(t+\Delta t)}{\partial d_{i}} \frac{S_{z \max }^{e *}}{d_{4}^{e} I_{z}^{e}}+\frac{\partial S_{z \max }^{e *}}{\partial d_{i}} \frac{\overline{\mathbf{F}}_{5}^{e}(t+\Delta t)}{d_{4}^{e} I_{z}^{e}} \\
& -\frac{\partial d_{4}^{e}}{\partial d_{i}} \frac{\overline{\mathbf{F}}_{5}^{e}(t+\Delta t) S_{z \max }^{e *}}{d_{4}^{e 2} I_{z}^{e}}-\frac{\partial I_{z}^{e}}{\partial d_{i}} \frac{\overline{\mathbf{F}}_{5}^{e}(t+\Delta t) S_{z \max }^{e *}}{d_{4}^{e} I_{z}^{e 2}}
\end{aligned}
$$

Differentiating Eq. (26) with respect to the design variable $d_{i}$, we have

$$
\begin{aligned}
& \frac{\partial \tau_{M x y}^{e}(t+\Delta t)}{\partial d_{i}}=\frac{\partial \overline{\mathbf{F}}_{M 2}^{e}(t+\Delta t)}{\partial d_{i}} \frac{S_{z \max }^{e *}}{d_{4}^{e} I_{z}^{e}}+\frac{\partial S_{z \max }^{e *}}{\partial d_{i}} \frac{\overline{\mathbf{F}}_{M 2}^{e}(t+\Delta t)}{d_{4}^{e} I_{z}^{e}} \\
& -\frac{\partial d_{4}^{e}}{\partial d_{i}} \frac{\overline{\mathbf{F}}_{M 2}^{e}(t+\Delta t) S_{z \max }^{e *}}{d_{4}^{e 2} I_{z}^{e}}-\frac{\partial I_{z}^{e}}{\partial d_{i}} \frac{\overline{\mathbf{F}}_{M 2}^{e}(t+\Delta t) S_{z \max }^{e *}}{d_{4}^{e} I_{z}^{e 2}}
\end{aligned}
$$




\subsubsection{Formulas for second derivatives of dynamic displacement and stress}

Now we will derive the formulas for the second derivatives of dynamic response, i.e., dynamic displacement and stress. Further differentiating Eq. (27) with respect to the design variable $d_{j}$, we have

$$
\mathbf{K}^{*} \frac{\partial^{2} \mathbf{x}(t+\Delta t)}{\partial d_{i} \partial d_{j}}=\frac{\partial^{2} \mathbf{F}^{*}(t+\Delta t)}{\partial d_{i} \partial d_{j}}-\frac{\partial^{2} \mathbf{K}^{*}}{\partial d_{i} \partial d_{j}} \mathbf{x}(t+\Delta t)-\frac{\partial \mathbf{K}^{*}}{\partial d_{i}} \frac{\partial \mathbf{x}(t+\Delta t)}{\partial d_{j}}-\frac{\partial \mathbf{K}^{*}}{\partial d_{j}} \frac{\partial \mathbf{x}(t+\Delta t)}{\partial d_{i}}
$$

where

$$
\frac{\partial^{2} \mathbf{K}^{*}}{\partial d_{i} \partial d_{j}}=\frac{\partial^{2} \mathbf{K}}{\partial d_{i} \partial d_{j}}+a_{0} \frac{\partial^{2} \mathbf{M}}{\partial d_{i} \partial d_{j}}+a_{1} \frac{\partial^{2} \mathbf{C}}{\partial d_{i} \partial d_{j}}
$$

and

$$
\begin{aligned}
& \frac{\partial^{2} \mathbf{F}^{*}(t+\Delta t)}{\partial d_{i} \partial d_{j}}=\frac{\partial^{2} \mathbf{M}}{\partial d_{i} \partial d_{j}}\left[a_{0} \mathbf{x}(t)+a_{2} \dot{\mathbf{x}}(t)+a_{3} \ddot{\mathbf{x}}(t)\right]+\frac{\partial \mathbf{M}}{\partial d_{i}}\left[a_{0} \frac{\partial \mathbf{x}(t)}{\partial d_{j}}+a_{2} \frac{\partial \dot{\mathbf{x}}(t)}{\partial d_{j}}+a_{3} \frac{\partial \ddot{\mathbf{x}}(t)}{\partial d_{j}}\right] \\
& +\frac{\partial \mathbf{M}}{\partial d_{j}}\left[a_{0} \frac{\partial \mathbf{x}(t)}{\partial d_{i}}+a_{2} \frac{\partial \dot{\mathbf{x}}(t)}{\partial d_{i}}+a_{3} \frac{\partial \ddot{\mathbf{x}}(t)}{\partial d_{i}}\right]+\mathbf{M}\left[a_{0} \frac{\partial^{2} \mathbf{x}(t)}{\partial d_{i} \partial d_{j}}+a_{2} \frac{\partial^{2} \dot{\mathbf{x}}(t)}{\partial d_{i} \partial d_{j}}+a_{3} \frac{\partial^{2} \ddot{\mathbf{x}}(t)}{\partial d_{i} \partial d_{j}}\right] \\
& +\frac{\partial^{2} \mathbf{C}}{\partial d_{i} \partial d_{j}}\left[a_{1} \mathbf{x}(t)+a_{4} \dot{\mathbf{x}}(t)+a_{5} \ddot{\mathbf{x}}(t)\right]+\frac{\partial \mathbf{C}}{\partial d_{i}}\left[a_{1} \frac{\partial \mathbf{x}(t)}{\partial d_{j}}+a_{4} \frac{\partial \dot{\mathbf{x}}(t)}{\partial d_{j}}+a_{5} \frac{\partial \ddot{\mathbf{x}}(t)}{\partial d_{j}}\right] \\
& +\frac{\partial \mathbf{C}}{\partial d_{j}}\left[a_{1} \frac{\partial \mathbf{x}(t)}{\partial d_{i}}+a_{4} \frac{\partial \dot{\mathbf{x}}(t)}{\partial d_{i}}+a_{5} \frac{\partial \ddot{\mathbf{x}}(t)}{\partial d_{i}}\right]+\mathbf{C}\left[a_{1} \frac{\partial^{2} \mathbf{x}(t)}{\partial d_{i} \partial d_{j}}+a_{4} \frac{\partial^{2} \dot{\mathbf{x}}(t)}{\partial d_{i} \partial d_{j}}+a_{5} \frac{\partial^{2} \ddot{\mathbf{x}}(t)}{\partial d_{i} \partial d_{j}}\right]
\end{aligned}
$$

After $\frac{\partial^{2} \mathbf{X}(t+\Delta t)}{\partial d_{i} \partial d_{j}}$ is computed from Eq. (40), with further differentiating Eq. (30) with respect to the design variables $d_{j}$, we have

$$
\frac{\partial^{2} \ddot{\mathbf{x}}(t+\Delta t)}{\partial d_{i} \partial d_{j}}=a_{0}\left[\frac{\partial^{2} \mathbf{x}(t+\Delta t)}{\partial d_{i} \partial d_{j}}-\frac{\partial^{2} \mathbf{x}(t)}{\partial d_{i} \partial d_{j}}\right]-a_{2} \frac{\partial^{2} \dot{\mathbf{x}}(t)}{\partial d_{i} \partial d_{j}}-a_{3} \frac{\partial^{2} \ddot{\mathbf{x}}(t)}{\partial d_{i} \partial d_{j}}
$$

Then $\frac{\partial^{2} \ddot{\mathbf{x}}(t+\Delta t)}{\partial d_{i} \partial d_{j}}$ is obtained from Eq. (43). Further differentiating Eq. (31) with respect to the design variables $d_{j}$, we obtain

$$
\frac{\partial^{2} \dot{\mathbf{x}}(t+\Delta t)}{\partial d_{i} \partial d_{j}}=\frac{\partial^{2} \dot{\mathbf{x}}(t)}{\partial d_{i} \partial d_{j}}+a_{6} \frac{\partial^{2} \ddot{\mathbf{x}}(t)}{\partial d_{i} \partial d_{j}}+a_{7} \frac{\partial^{2} \ddot{\mathbf{x}}(t+\Delta t)}{\partial d_{i} \partial d_{j}}
$$

Further differentiating Eq. (32) with respect to the design variable $d_{j}$, we have

$$
\begin{aligned}
& \frac{\partial^{2} \overline{\mathbf{F}}^{e}(t+\Delta t)}{\partial d_{i} \partial d_{j}}=\frac{\partial^{2} \overline{\mathbf{K}}^{e}}{\partial d_{i} \partial d_{j}} \mathbf{T}^{e} \delta^{e}(t+\Delta t)+\frac{\partial \overline{\mathbf{K}}^{e}}{\partial d_{i}} \mathbf{T}^{e} \frac{\partial \delta^{e}(t+\Delta t)}{\partial d_{j}} \\
& +\frac{\partial \overline{\mathbf{K}}^{e}}{\partial d_{j}} \mathbf{T}^{e} \frac{\partial \delta^{e}(t+\Delta t)}{\partial d_{i}}+\overline{\mathbf{K}}^{e} \mathbf{T}^{e} \frac{\partial^{2} \delta^{e}(t+\Delta t)}{\partial d_{i} \partial d_{j}}
\end{aligned}
$$

Further differentiating Eq. (33) with respect to the design variable $d_{j}$, we obtain

$$
\frac{\partial^{2} \overline{\mathbf{F}}_{M}^{e}(t+\Delta t)}{\partial d_{i} \partial d_{j}}=\left[\begin{array}{lll}
-\frac{\partial^{2} \overline{\mathbf{F}}_{1}^{e}(t+\Delta t)}{\partial d_{i} \partial d_{j}} & -\frac{\partial^{2} \overline{\mathbf{F}}_{2}^{e}(t+\Delta t)}{\partial d_{i} \partial d_{j}} & \frac{l_{e}}{2} \frac{\partial^{2} \overline{\mathbf{F}}_{2}^{e}(t+\Delta t)}{\partial d_{i} \partial d_{j}}-\frac{\partial^{2} \overline{\mathbf{F}}_{3}^{e}(t+\Delta t)}{\partial d_{i} \partial d_{j}}
\end{array}\right]^{T}
$$

Further differentiating Eq. (34) with respect to the design variable $d_{j}$, we have

$$
\begin{aligned}
& \frac{\partial^{2} \sigma_{i x}^{e}(t+\Delta t)}{\partial d_{i} \partial d_{j}}=\frac{\partial^{2} \overline{\mathbf{F}}_{1}^{e}(t+\Delta t)}{\partial d_{i} \partial d_{j}} \frac{1}{A^{e}}-\frac{1}{A^{e 2}} \frac{\partial \overline{\mathbf{F}}_{1}^{e}(t+\Delta t)}{\partial d_{i}} \frac{\partial A^{e}}{\partial d_{j}}-\frac{1}{A^{e 2}} \frac{\partial \overline{\mathbf{F}}_{1}^{e}(t+\Delta t)}{\partial d_{j}} \frac{\partial A^{e}}{\partial d_{i}} \\
& +\frac{2 \overline{\mathbf{F}}_{1}^{e}(t+\Delta t)}{A^{e 3}} \frac{\partial A^{e}}{\partial d_{j}} \frac{\partial A^{e}}{\partial d_{i}}-\frac{\overline{\mathbf{F}}_{1}^{e}(t+\Delta t)}{A^{e 2}} \frac{\partial^{2} A^{e}}{\partial d_{i} \partial d_{j}}+\frac{\partial^{2} \overline{\mathbf{F}}_{3}^{e}(t+\Delta t)}{\partial d_{i} \partial d_{j}} \frac{y_{m}^{e}}{E I_{z}^{e}}+\frac{\partial \overline{\mathbf{F}}_{3}^{e}(t+\Delta t)}{\partial d_{i}} \frac{\partial y_{m}^{e}}{\partial d_{j}} \frac{1}{E I_{z}^{e}} \\
& -\frac{\partial \overline{\mathbf{F}}_{3}^{e}(t+\Delta t)}{\partial d_{i}} \frac{y_{m}^{e}}{E I_{z}^{e 2}} \frac{\partial I_{z}^{e}}{\partial d_{j}}+\frac{\partial \overline{\mathbf{F}}_{3}^{e}(t+\Delta t)}{\partial d_{j}} \frac{1}{E I_{z}^{e}} \frac{\partial y_{m}^{e}}{\partial d_{i}}-\frac{\partial I_{z}^{e}}{\partial d_{j}} \frac{\overline{\mathbf{F}}_{3}^{e}(t+\Delta t)}{E I_{z}^{e 2}} \frac{\partial y_{m}^{e}}{\partial d_{i}}+\frac{\overline{\mathbf{F}}_{3}^{e}(t+\Delta t)}{E I_{z}^{e}} \frac{\partial^{2} y_{m}^{e}}{\partial d_{i} \partial d_{j}} \\
& -\frac{\partial \overline{\mathbf{F}}_{3}^{e}(t+\Delta t)}{\partial d_{j}} \frac{y_{m}^{e}}{E I_{z}^{e 2}} \frac{\partial I_{z}^{e}}{\partial d_{i}}-\frac{\partial y_{m}^{e}}{\partial d_{j}} \frac{\overline{\mathbf{F}}_{3}^{e}(t+\Delta t)}{E I_{z}^{e 2}} \frac{\partial I_{z}^{e}}{\partial d_{i}}+\frac{\partial I_{z}^{e}}{\partial d_{j}} \frac{2 \overline{\mathbf{F}}_{3}^{e}(t+\Delta t) y_{m}^{e}}{E I_{z}^{e 3}} \frac{\partial I_{z}^{e}}{\partial d_{i}}-\frac{\overline{\mathbf{F}}_{3}^{e}(t+\Delta t) y_{m}^{e}}{E I_{z}^{e 2}} \frac{\partial^{2} I_{z}^{e}}{\partial d_{i} \partial d_{j}}
\end{aligned}
$$


Further differentiating Eq. (35) with respect to the design variable $d_{j}$, we obtain

$$
\begin{aligned}
& \frac{\partial^{2} \sigma_{j x}^{e}(t+\Delta t)}{\partial d_{i} \partial d_{j}}=\frac{\partial^{2} \overline{\mathbf{F}}_{4}^{e}(t+\Delta t)}{\partial d_{i} \partial d_{j}} \frac{1}{A^{e}}-\frac{1}{A^{e 2}} \frac{\partial \overline{\mathbf{F}}_{4}^{e}(t+\Delta t)}{\partial d_{i}} \frac{\partial A^{e}}{\partial d_{j}}-\frac{1}{A^{e 2}} \frac{\partial \overline{\mathbf{F}}_{4}^{e}(t+\Delta t)}{\partial d_{j}} \frac{\partial A^{e}}{\partial d_{i}} \\
& +\frac{2 \overline{\mathbf{F}}_{4}^{e}(t+\Delta t)}{A^{e 3}} \frac{\partial A^{e}}{\partial d_{j}} \frac{\partial A^{e}}{\partial d_{i}}-\frac{\overline{\mathbf{F}}_{4}^{e}(t+\Delta t)}{A^{e 2}} \frac{\partial^{2} A^{e}}{\partial d_{i} \partial d_{j}}+\frac{\partial^{2} \overline{\mathbf{F}}_{6}^{e}(t+\Delta t)}{\partial d_{i} \partial d_{j}} \frac{y_{m}^{e}}{E I_{z}^{e}}+\frac{\partial \overline{\mathbf{F}}_{6}^{e}(t+\Delta t)}{\partial d_{i}} \frac{\partial y_{m}^{e}}{\partial d_{j}} \frac{1}{E I_{z}^{e}} \\
& -\frac{\partial \overline{\mathbf{F}}_{6}^{e}(t+\Delta t)}{\partial d_{i}} \frac{y_{m}^{e}}{E I_{z}^{e 2}} \frac{\partial I_{z}^{e}}{\partial d_{j}}+\frac{\partial \overline{\mathbf{F}}_{6}^{e}(t+\Delta t)}{\partial d_{j}} \frac{1}{E I_{z}^{e}} \frac{\partial y_{m}^{e}}{\partial d_{i}}-\frac{\partial I_{z}^{e}}{\partial d_{j}} \frac{\overline{\mathbf{F}}_{6}^{e}(t+\Delta t)}{E I_{z}^{e 2}} \frac{\partial y_{m}^{e}}{\partial d_{i}}+\frac{\overline{\mathbf{F}}_{6}^{e}(t+\Delta t)}{E I_{z}^{e}} \frac{\partial^{2} y_{m}^{e}}{\partial d_{i} \partial d_{j}} \\
& -\frac{\partial \overline{\mathbf{F}}_{6}^{e}(t+\Delta t)}{\partial d_{j}} \frac{y_{m}^{e}}{E I_{z}^{e 2}} \frac{\partial I_{z}^{e}}{\partial d_{i}}-\frac{\partial y_{m}^{e}}{\partial d_{j}} \frac{\overline{\mathbf{F}}_{6}^{e}(t+\Delta t)}{E I_{z}^{e 2}} \frac{\partial I_{z}^{e}}{\partial d_{i}}+\frac{\partial I_{z}^{e}}{\partial d_{j}} \frac{2 \overline{\mathbf{F}}_{6}^{e}(t+\Delta t) y_{m}^{e}}{E I_{z}^{e 3}} \frac{\partial I_{z}^{e}}{\partial d_{i}}-\frac{\overline{\mathbf{F}}_{6}^{e}(t+\Delta t) y_{m}^{e}}{E I_{z}^{e 2}} \frac{\partial^{2} I_{z}^{e}}{\partial d_{i} \partial d_{j}}
\end{aligned}
$$

Further differentiating Eq. (36) with respect to the design variable $d_{j}$, we have

$$
\begin{aligned}
& \frac{\partial^{2} \sigma_{M x}^{e}(t+\Delta t)}{\partial d_{i} \partial d_{j}}=\frac{\partial^{2} \overline{\mathbf{F}}_{M 1}^{e}(t+\Delta t)}{\partial d_{i} \partial d_{j}} \frac{1}{A^{e}}-\frac{1}{A^{e 2}} \frac{\partial \overline{\mathbf{F}}_{M 1}^{e}(t+\Delta t)}{\partial d_{i}} \frac{\partial A^{e}}{\partial d_{j}}-\frac{1}{A^{e 2}} \frac{\partial \overline{\mathbf{F}}_{M 1}^{e}(t+\Delta t)}{\partial d_{j}} \frac{\partial A^{e}}{\partial d_{i}} \\
& +\frac{2 \overline{\mathbf{F}}_{M 1}^{e}(t+\Delta t)}{A^{e 3}} \frac{\partial A^{e}}{\partial d_{j}} \frac{\partial A^{e}}{\partial d_{i}}-\frac{\overline{\mathbf{F}}_{M 1}^{e}(t+\Delta t)}{A^{e 2}} \frac{\partial^{2} A^{e}}{\partial d_{i} \partial d_{j}}+\frac{\partial^{2} \overline{\mathbf{F}}_{M 3}^{e}(t+\Delta t)}{\partial d_{i} \partial d_{j}} \frac{y_{m}^{e}}{E I_{z}^{e}}+\frac{\partial \overline{\mathbf{F}}_{M 3}^{e}(t+\Delta t)}{\partial d_{i}} \frac{\partial y_{m}^{e}}{\partial d_{j}} \frac{1}{E I_{z}^{e}} \\
& -\frac{\partial \overline{\mathbf{F}}_{M 3}^{e}(t+\Delta t)}{\partial d_{i}} \frac{y_{m}^{e}}{E I_{z}^{e 2}} \frac{\partial I_{z}^{e}}{\partial d_{j}}+\frac{\partial \overline{\mathbf{F}}_{M 3}^{e}(t+\Delta t)}{\partial d_{j}} \frac{1}{E I_{z}^{e}} \frac{\partial y_{m}^{e}}{\partial d_{i}}-\frac{\partial I_{z}^{e}}{\partial d_{j}} \frac{\overline{\mathbf{F}}_{M 3}^{e}(t+\Delta t)}{E I_{z}^{e 2}} \frac{\partial y_{m}^{e}}{\partial d_{i}}+\frac{\overline{\mathbf{F}}_{M 3}^{e}(t+\Delta t)}{E I_{z}^{e}} \frac{\partial^{2} y_{m}^{e}}{\partial d_{i} \partial d_{j}} \\
& -\frac{\partial \overline{\mathbf{F}}_{M 3}^{e}(t+\Delta t)}{\partial d_{j}} \frac{y_{m}^{e}}{E I_{z}^{e 2}} \frac{\partial I_{z}^{e}}{\partial d_{i}}-\frac{\partial y_{m}^{e}}{\partial d_{j}} \frac{\overline{\mathbf{F}}_{M 3}^{e}(t+\Delta t)}{E I_{z}^{e 2}} \frac{\partial I_{z}^{e}}{\partial d_{i}} \\
& +\frac{\partial I_{z}^{e}}{\partial d_{j}} \frac{2 \overline{\mathbf{F}}_{M 3}^{e}(t+\Delta t) y_{m}^{e}}{E I_{z}^{e 3}} \frac{\partial I_{z}^{e}}{\partial d_{i}}-\frac{\overline{\mathbf{F}}_{M 3}^{e}(t+\Delta t) y_{m}^{e}}{E I_{z}^{e 2}} \frac{\partial^{2} I_{z}^{e}}{\partial d_{i} \partial d_{j}}
\end{aligned}
$$

Further differentiating Eq. (37) with respect to the design variable $d_{j}$, we obtain

$$
\begin{aligned}
& \frac{\partial^{2} \tau_{i x y}^{e}(t+\Delta t)}{\partial d_{i} \partial d_{j}}=\frac{\partial^{2} \overline{\mathbf{F}}_{2}^{e}(t+\Delta t)}{\partial d_{i} \partial d_{j}} \frac{S_{z \max }^{e *}}{d_{4}^{e} I_{z}^{e}}+\frac{\partial \overline{\mathbf{F}}_{2}^{e}(t+\Delta t)}{\partial d_{i}} \frac{\partial S_{z \max }^{e *}}{\partial d_{j}} \frac{1}{d_{4}^{e} I_{z}^{e}}-\frac{\partial \overline{\mathbf{F}}_{2}^{e}(t+\Delta t)}{\partial d_{i}} \frac{\partial d_{4}^{e}}{\partial d_{j}} \frac{S_{z \max }^{e *}}{d_{4}^{e 2} I_{z}^{e}} \\
& -\frac{\partial \overline{\mathbf{F}}_{2}^{e}(t+\Delta t)}{\partial d_{i}} \frac{\partial I_{z}^{e}}{\partial d_{j}} \frac{S_{z \max }^{e *}}{d_{4}^{e} I_{z}^{e 2}}+\frac{\partial^{2} S_{z \max }^{e *}}{\partial d_{i} \partial d_{j}} \frac{\overline{\mathbf{F}}_{2}^{e}(t+\Delta t)}{d_{4}^{e} I_{z}^{e}}+\frac{\partial S_{z \max }^{e *}}{\partial d_{i}} \frac{\partial \overline{\mathbf{F}}_{2}^{e}(t+\Delta t)}{\partial d_{j}} \frac{1}{d_{4}^{e} I_{z}^{e}} \\
& -\frac{\partial S_{z \max }^{e *}}{\partial d_{i}} \frac{\partial d_{4}^{e}}{\partial d_{j}} \frac{\overline{\mathbf{F}}_{2}^{e}}{d_{4}^{e}(t+\Delta t)} \frac{\partial S_{z \max }^{e *}}{\partial d_{i}} \frac{\partial I_{z}^{e}}{\partial d_{j}} \frac{\overline{\mathbf{F}}_{2}^{e}(t+\Delta t)}{d_{4}^{e} I_{z}^{e}}-\frac{\partial^{2} d_{4}^{e}}{\partial d_{i} \partial d_{j}^{e}} \frac{\overline{\mathbf{F}}_{2}^{e}(t+\Delta t) S_{z \max }^{e *}}{d_{4}^{e 2} I_{z}^{e}} \\
& -\frac{\partial d_{4}^{e}}{\partial d_{i}} \frac{\partial \overline{\mathbf{F}}_{2}^{e}(t+\Delta t)}{\partial d_{j}} \frac{S_{z \max }^{e *}}{d_{4}^{e 2} I_{z}^{e}}-\frac{\partial d_{4}^{e}}{\partial d_{i}} \frac{\partial S_{z \max }^{e *}}{\partial d_{j}} \frac{\overline{\mathbf{F}}_{2}^{e}(t+\Delta t)}{d_{4}^{e 2} I_{z}^{e}}+\frac{\partial d_{4}^{e}}{\partial d_{i}} \frac{\partial d_{4}^{e}}{\partial d_{j}^{e}} \frac{2 \overline{\mathbf{F}}_{2}^{e}(t+\Delta t) S_{z \max }^{e *}}{d_{4}^{e 3} I_{z}^{e}} \\
& +\frac{\partial d_{4}^{e}}{\partial d_{i}} \frac{\partial I_{z}^{e}}{\partial d_{j}} \frac{\overline{\mathbf{F}}_{2}^{e}(t+\Delta t) S_{z \max }^{e *}}{d_{4}^{e 2} I_{z}^{e 2}}-\frac{\partial^{2} I_{z}^{e}}{\partial d_{i} \partial d_{j}} \frac{\overline{\mathbf{F}}_{2}^{e}(t+\Delta t) S_{z \max }^{e *}}{d_{4}^{e} I_{z}^{e 2}}-\frac{\partial I_{z}^{e}}{\partial d_{i}} \frac{\partial \overline{\mathbf{F}}_{2}^{e}(t+\Delta t)}{\partial d_{j}} \frac{S_{z \max }^{e *}}{d_{4}^{e} I_{z}^{2}} \\
& -\frac{\partial I_{z}^{e}}{\partial d_{i}} \frac{\partial S_{z \max }^{e *}}{\partial d_{j}} \frac{\overline{\mathbf{F}}_{2}^{e}(t+\Delta t)}{d_{4}^{e} I_{z}^{e 2}}+\frac{\partial I_{z}^{e}}{\partial d_{i}} \frac{\partial d_{4}^{e}}{\partial d_{j}} \frac{\overline{\mathbf{F}}_{2}^{e}(t+\Delta t) S_{z \max }^{e *}}{d_{4}^{e 2} I_{z}^{e 2}}+\frac{\partial I_{z}^{e}}{\partial d_{i}} \frac{\partial I_{z}^{e}}{\partial d_{j}} \frac{2 \overline{\mathbf{F}}_{2}^{e}(t+\Delta t) S_{z \max }^{e *}}{d_{4}^{e} I_{z}^{e 3}}
\end{aligned}
$$

Further differentiating Eq. (38) with respect to the design variable $d_{j}$, we have

$$
\begin{aligned}
& \frac{\partial^{2} \tau_{j x y}^{e}(t+\Delta t)}{\partial d_{i} \partial d_{j}}=\frac{\partial^{2} \overline{\mathbf{F}}_{5}^{e}(t+\Delta t)}{\partial d_{i} \partial d_{j}} \frac{S_{z \max }^{e *}}{d_{4}^{e} I_{z}^{e}}+\frac{\partial \overline{\mathbf{F}}_{5}^{e}(t+\Delta t)}{\partial d_{i}} \frac{\partial S_{z \max }^{e *}}{\partial d_{j}} \frac{1}{d_{4}^{e} I_{z}^{e}}-\frac{\partial \overline{\mathbf{F}}_{5}^{e}(t+\Delta t)}{\partial d_{i}} \frac{\partial d_{4}^{e}}{\partial d_{j}} \frac{S_{z \max }^{e *}}{d_{4}^{e 2} I_{z}^{e}} \\
& -\frac{\partial \overline{\mathbf{F}}_{5}^{e}(t+\Delta t)}{\partial d_{i}} \frac{\partial I_{z}^{e}}{\partial d_{j}} \frac{S_{z \max }^{e *}}{d_{4}^{e} I_{z}^{e 2}}+\frac{\partial^{2} S_{z \max }^{e *}}{\partial d_{i} \partial d_{j}} \frac{\overline{\mathbf{F}}_{5}^{e}(t+\Delta t)}{d_{4}^{e} I_{z}^{e}}+\frac{\partial S_{z \max }^{e *}}{\partial d_{i}} \frac{\partial \overline{\mathbf{F}}_{5}^{e}(t+\Delta t)}{\partial d_{j}} \frac{1}{d_{4}^{e} I_{z}^{e}}
\end{aligned}
$$




$$
\begin{aligned}
& -\frac{\partial S_{z \max }^{e *}}{\partial d_{i}} \frac{\partial d_{4}^{e}}{\partial d_{j}} \frac{\overline{\mathbf{F}}_{5}^{e}(t+\Delta t)}{d_{4}^{e 2} I_{z}^{e}}-\frac{\partial S_{z \max }^{e *}}{\partial d_{i}} \frac{\partial I_{z}^{e}}{\partial d_{j}} \frac{\overline{\mathbf{F}}_{5}^{e}(t+\Delta t)}{d_{4}^{e} I_{z}^{e 2}}-\frac{\partial^{2} d_{4}^{e}}{\partial d_{i} \partial d_{j}} \frac{\overline{\mathbf{F}}_{5}^{e}(t+\Delta t) S_{z \max }^{e *}}{d_{4}^{e 2} I_{z}^{e}} \\
& -\frac{\partial d_{4}^{e}}{\partial d_{i}} \frac{\partial \overline{\mathbf{F}}_{5}^{e}(t+\Delta t)}{\partial d_{j}} \frac{S_{z \max }^{e *}}{d_{4}^{e 2} I_{z}^{e}}-\frac{\partial d_{4}^{e}}{\partial d_{i}} \frac{\partial S_{z \max }^{e *}}{\partial d_{j}} \frac{\overline{\mathbf{F}}_{5}^{e}(t+\Delta t)}{d_{4}^{e 2} I_{z}^{e}}+\frac{\partial d_{4}^{e}}{\partial d_{i}} \frac{\partial d_{4}^{e}}{\partial d_{j}} \frac{2 \overline{\mathbf{F}}_{5}^{e}(t+\Delta t) S_{z \max }^{e *}}{d_{4}^{e 3} I_{z}^{e}} \\
& +\frac{\partial d_{4}^{e}}{\partial d_{i}} \frac{\partial I_{z}^{e}}{\partial d_{j}} \frac{\overline{\mathbf{F}}_{5}^{e}(t+\Delta t) S_{z \max }^{e *}}{d_{4}^{e 2} I_{z}^{e 2}}-\frac{\partial^{2} I_{z}^{e}}{\partial d_{i} \partial d_{j}} \frac{\overline{\mathbf{F}}_{5}^{e}(t+\Delta t) S_{z \max }^{e *}}{d_{4}^{e} I_{z}^{e 2}}-\frac{\partial I_{z}^{e}}{\partial d_{i}} \frac{\partial \overline{\mathbf{F}}_{5}^{e}(t+\Delta t)}{\partial d_{j}} \frac{S_{z \max }^{e *}}{d_{4}^{e} I_{z}^{e 2}} \\
& -\frac{\partial I_{z}^{e}}{\partial d_{i}} \frac{\partial S_{z \max }^{e *}}{\partial d_{j}} \frac{\overline{\mathbf{F}}_{5}^{e}(t+\Delta t)}{d_{4}^{e} I_{z}^{e 2}}+\frac{\partial I_{z}^{e}}{\partial d_{i}} \frac{\partial d_{4}^{e}}{\partial d_{j}} \frac{\overline{\mathbf{F}}_{5}^{e}(t+\Delta t) S_{z \max }^{e *}}{d_{4}^{e 2} I_{z}^{e 2}}+\frac{\partial I_{z}^{e}}{\partial d_{i}} \frac{\partial I_{z}^{e}}{\partial d_{j}} \frac{2 \overline{\mathbf{F}}_{5}^{e}(t+\Delta t) S_{z \max }^{e *}}{d_{4}^{e} I_{z}^{e 3}}
\end{aligned}
$$

Further differentiating Eq. (39) with respect to the design variable $d_{j}$, we obtain

$$
\begin{aligned}
& \frac{\partial^{2} \tau_{M x y}^{e}(t+\Delta t)}{\partial d_{i} \partial d_{j}}=\frac{\partial^{2} \overline{\mathbf{F}}_{M 2}^{e}(t+\Delta t)}{\partial d_{i} \partial d_{j}} \frac{S_{z \max }^{e *}}{d_{4}^{e} I_{z}^{e}}+\frac{\partial \overline{\mathbf{F}}_{M 2}^{e}(t+\Delta t)}{\partial d_{i}} \frac{\partial S_{z \max }^{e *}}{\partial d_{j}} \frac{1}{d_{4}^{e} I_{z}^{e}}-\frac{\partial \overline{\mathbf{F}}_{M 2}^{e}(t+\Delta t)}{\partial d_{i}} \frac{\partial d_{4}^{e}}{\partial d_{j}} \frac{S_{z \max }^{e *}}{d_{4}^{e 2} I_{z}^{e}} \\
& -\frac{\partial \overline{\mathbf{F}}_{M 2}^{e}(t+\Delta t)}{\partial d_{i}} \frac{\partial I_{z}^{e}}{\partial d_{j}} \frac{S_{z \max }^{e *}}{d_{4}^{e} I_{z}^{e 2}}+\frac{\partial^{2} S_{z \max }^{e *}}{\partial d_{i} \partial d_{j}} \frac{\overline{\mathbf{F}}_{M 2}^{e}(t+\Delta t)}{d_{4}^{e} I_{z}^{e}}+\frac{\partial S_{z \max }^{e *}}{\partial d_{i}} \frac{\partial \overline{\mathbf{F}}_{M 2}^{e}(t+\Delta t)}{\partial d_{j}} \frac{1}{d_{4}^{e} I_{z}^{e}} \\
& -\frac{\partial S_{z \max }^{e *}}{\partial d_{i}} \frac{\partial d_{4}^{e}}{\partial d_{j}} \frac{\overline{\mathbf{F}}_{M 2}^{e}(t+\Delta t)}{d_{4}^{e 2} I_{z}^{e}}-\frac{\partial S_{z \max }^{e *}}{\partial d_{i}} \frac{\partial I_{z}^{e}}{\partial d_{j}} \frac{\overline{\mathbf{F}}_{M 2}^{e}(t+\Delta t)}{d_{4}^{e} I_{z}^{e 2}}-\frac{\partial^{2} d_{4}^{e}}{\partial d_{i} \partial d_{j}} \frac{\overline{\mathbf{F}}_{M 2}^{e}(t+\Delta t) S_{z \max }^{e *}}{d_{4}^{e 2} I_{z}^{e}} \\
& -\frac{\partial d_{4}^{e}}{\partial d_{i}} \frac{\partial \overline{\mathbf{F}}_{M 2}^{e}(t+\Delta t)}{\partial d_{j}} \frac{S_{z \max }^{e *}}{d_{4}^{e 2} I_{z}^{e}}-\frac{\partial d_{4}^{e}}{\partial d_{i}} \frac{\partial S_{z \max }^{e *}}{\partial d_{j}} \frac{\overline{\mathbf{F}}_{M 2}^{e}(t+\Delta t)}{d_{4}^{e 2} I_{z}^{e}}+\frac{\partial d_{4}^{e}}{\partial d_{i}} \frac{\partial d_{4}^{e}}{\partial d_{j}} \frac{2 \overline{\mathbf{F}}_{M 2}^{e}(t+\Delta t) S_{z \max }^{e *}}{d_{4}^{e 3} I_{z}^{e}} \\
& +\frac{\partial d_{4}^{e}}{\partial d_{i}} \frac{\partial I_{z}^{e}}{\partial d_{j}^{e}} \frac{\overline{\mathbf{F}}_{M 2}^{e}(t+\Delta t) S_{z \max }^{e *}}{d_{4}^{e 2} I_{z}^{e 2}}-\frac{\partial^{2} I_{z}^{e}}{\partial d_{i} \partial d_{j}} \frac{\overline{\mathbf{F}}_{M 2}^{e}(t+\Delta t) S_{z \max }^{e *}}{d_{4}^{e} I_{z}^{e 2}}-\frac{\partial I_{z}^{e}}{\partial d_{i}} \frac{\partial \overline{\mathbf{F}}_{M 2}^{e}(t+\Delta t)}{\partial d_{j}} \frac{S_{z \max }^{e *}}{d_{4}^{e} I_{z}^{e 2}} \\
& -\frac{\partial I_{z}^{e}}{\partial d_{i}} \frac{\partial S_{z \max }^{e *}}{\partial d_{j}} \frac{\overline{\mathbf{F}}_{M 2}^{e}(t+\Delta t)}{d_{4}^{e} I_{z}^{e 2}}+\frac{\partial I_{z}^{e}}{\partial d_{i}} \frac{\partial d_{4}^{e}}{\partial d_{j}} \frac{\overline{\mathbf{F}}_{M 2}^{e}(t+\Delta t) S_{z \max }^{e *}}{d_{4}^{e 2} I_{z}^{e 2}}+\frac{\partial I_{z}^{e}}{\partial d_{i}} \frac{\partial I_{z}^{e}}{\partial d_{j}} \frac{2 \overline{\mathbf{F}}_{M 2}^{e}(t+\Delta t) S_{z \max }^{e *}}{d_{4}^{e} I_{z}^{e 3}}
\end{aligned}
$$

\subsubsection{Computation procedure of the first and second derivatives of dynamic displacement and stress}

In this work, we suppose that the initial conditions are $\mathbf{x}(0)=0, \dot{\mathbf{x}}(0)=0, \ddot{\mathbf{x}}(0)=0$, and the dynamic responses (nodal displacements and stresses), their first and second derivatives with respect to design variables are equal to zero.

Section 2.1.2 gives the formulas for calculating the first and second derivatives of the dynamic response. This section provides the detailed computation procedure as follows.

Procedure of calculating dynamic response first and second derivatives:

\section{Step 1 Initial calculations:}

Step $1.1 \mathbf{x}(0)=0, \dot{\mathbf{x}}(0)=0, \ddot{\mathbf{x}}(0)=0, \sigma_{i x}^{e}(0)=0, \sigma_{j x}^{e}(0)=0, \sigma_{M x}^{e}(0)=0, \tau_{i x y}^{e}(0)=0, \tau_{j x y}^{e}(0)=0$,

$$
\tau_{M x y}^{e}(0)=0 \text {. }
$$

Step $1.2 \frac{\partial \mathbf{X}(0)}{\partial d_{i}}=0, \frac{\partial \dot{\mathbf{X}}(0)}{\partial d_{i}}=0, \frac{\partial \ddot{\mathbf{X}}(0)}{\partial d_{i}}=0, \frac{\partial \sigma_{\sigma_{x}}^{e}(0)}{\partial d_{i}}=0, \frac{\partial \sigma_{j x}^{e}(0)}{\partial d_{i}}=0, \frac{\partial \sigma_{M x}^{e}(0)}{\partial d_{i}}=0, \frac{\partial \tau_{i x y}^{e}(0)}{\partial d_{i}}=0$,

$$
\frac{\partial \tau_{j x y}^{e}(0)}{\partial d_{i}}=0, \frac{\partial \tau_{M x y}^{e}(0)}{\partial d_{i}}=0
$$

Step $1.3 \frac{\partial^{2} \mathbf{X}(0)}{\partial d_{i} \partial d_{j}}=0, \frac{\partial^{2} \dot{\mathbf{X}}(0)}{\partial d_{i} \partial d_{j}}=0, \frac{\partial^{2} \ddot{\mathbf{x}}(0)}{\partial d_{i} \partial d_{j}}=0, \frac{\partial^{2} \sigma_{i x}^{e}(0)}{\partial d_{i} \partial d_{j}}=0, \frac{\partial^{2} \sigma_{j x}^{e}(0)}{\partial d_{i} \partial d_{j}}=0, \frac{\partial^{2} \sigma_{M x}^{e}(0)}{\partial d_{i} \partial d_{j}}=0$,

$$
\frac{\partial^{2} \tau_{i x y}^{e}(0)}{\partial d_{i} \partial d_{j}}=0, \frac{\partial^{2} \tau_{j x y}^{e}(0)}{\partial d_{i} \partial d_{j}}=0, \frac{\partial^{2} \tau_{M x y}^{e}(0)}{\partial d_{i} \partial d_{j}}=0 .
$$


Step 1.4 Set $\Delta t$.

Step 1.5 Compute integral constants:

$$
\begin{aligned}
& a_{0}=\frac{1}{\beta \Delta t^{2}}, a_{1}=\frac{\delta}{\beta \Delta t}, a_{2}=\frac{1}{\beta \Delta t}, a_{3}=\frac{1}{2 \beta}-1, a_{4}=\frac{\delta}{\beta}-1, a_{5}=\frac{\Delta t}{2}\left(\frac{\delta}{\beta}-2\right), a_{6}=\Delta t(1-\delta), \\
& a_{7}=\delta \Delta t .
\end{aligned}
$$

where integral parameters $\delta \geqslant 0.5$ and $\beta=0.25(0.5+\delta)^{2}$.

Step 1.6 Solve Eq. (15) $\Rightarrow \mathbf{K}^{*}$.

\section{Step 2 Calculations for each time step $t+\Delta t$ :}

Step 2.1 Solve Eq. (14) $\Rightarrow \mathbf{x}(t+\Delta t)$

Step 2.2 Solve Eq. (17) $\Rightarrow \ddot{\mathbf{x}}(t+\Delta t)$, solve Eq. $(21) \Rightarrow \sigma_{i x}^{e}(t+\Delta t)$, solve Eq. (22) $\Rightarrow \sigma_{j x}^{e}(t+\Delta t)$, solve Eq. (23) $\Rightarrow \sigma_{M x}^{e}(t+\Delta t)$, solve Eq. (24) $\Rightarrow \tau_{i x y}^{e}(t+\Delta t)$, solve Eq. $(25) \Rightarrow \tau_{j x y}^{e}(t+\Delta t)$, and solve Eq. $(26) \Rightarrow \tau_{M x y}^{e}(t+\Delta t)$.

Step 2.3 Solve Eq. $(18) \Rightarrow \dot{\mathbf{x}}(t+\Delta t)$

Step 2.4. Solve Eq. (27) $\Rightarrow \frac{\partial \mathbf{x}(t+\Delta t)}{\partial d_{i}}$.

Step 2.5 Solve Eq. $(30) \Rightarrow \frac{\partial \ddot{\mathbf{x}}(t+\Delta t)}{\partial d_{i}}$, solve Eq. (34) $\Rightarrow \frac{\partial \sigma_{i x}^{e}(t+\Delta t)}{\partial d_{i}}$,

solve Eq. $(35) \Rightarrow \frac{\partial \sigma_{j x}^{e}(t+\Delta t)}{\partial d_{i}}$, solve Eq. $(36) \Rightarrow \frac{\partial \sigma_{M x}^{e}(t+\Delta t)}{\partial d_{i}}$,

solve Eq. $(37) \Rightarrow \frac{\partial \tau_{i x y}^{e}(t+\Delta t)}{\partial d_{i}}$, solve Eq. $(38) \Rightarrow \frac{\partial \tau_{j x y}^{e}(t+\Delta t)}{\partial d_{i}}$,

and solve Eq. (39) $\Rightarrow \frac{\partial \tau_{M x y}^{e}(t+\Delta t)}{\partial d_{i}}$.

Step 2.6 Solve Eq. $(31) \Rightarrow \frac{\partial \dot{\mathbf{X}}(t+\Delta t)}{\partial d_{i}}$.

Step 2.7 Solve Eq. $(40) \Rightarrow \frac{\partial^{2} \mathbf{X}(t+\Delta t)}{\partial d_{i} \partial d_{j}}$.

Step 2.8 Solve Eq. (43) $\Rightarrow \frac{\partial^{2} \ddot{\mathbf{x}}(t+\Delta t)}{\partial d_{i} \partial d_{j}}$, solve Eq. (47) $\Rightarrow \frac{\partial^{2} \sigma_{i x}^{e}(t+\Delta t)}{\partial d_{i} \partial d_{j}}$,

solve Eq. (48) $\Rightarrow \frac{\partial^{2} \sigma_{j x}^{e}(t+\Delta t)}{\partial d_{i} \partial d_{j}}$, solve Eq. (49) $\Rightarrow \frac{\partial^{2} \sigma_{M x}^{e}(t+\Delta t)}{\partial d_{i} \partial d_{j}}$,

solve Eq. $(50) \Rightarrow \frac{\partial^{2} \tau_{i x y}^{e}(t+\Delta t)}{\partial d_{i} \partial d_{j}}$, solve Eq. $(51) \Rightarrow \frac{\partial^{2} \tau_{j x y}^{e}(t+\Delta t)}{\partial d_{i} \partial d_{j}}$,

and solve Eq. $(52) \Rightarrow \frac{\partial^{2} \tau_{M x y}^{e}(t+\Delta t)}{\partial d_{i} \partial d_{j}}$.

Step 2.9 Solve Eq. (44) $\Rightarrow \frac{\partial^{2} \dot{\mathbf{x}}(t+\Delta t)}{\partial d_{i} \partial d_{j}}$.

Step 3 Repetition for the next time step. Replace $t$ by $t+\Delta t$ and implement steps 2.1 to 2.9 for the next time step.

\subsection{First and second derivatives of structural mass}

We use structural mass as the objective function in optimization. The objective function or the structural mass can be expressed as

$$
w(\mathbf{d})=\sum_{e=1}^{N} \rho A^{e} l_{e}
$$

The first derivatives of the structural mass are obtained by differentiating Eq. (53) with respect to the design variables,

$$
\frac{\partial w(\mathbf{d})}{\partial d_{i}}=\sum_{e=1}^{N} \rho l_{e} \frac{\partial A^{e}}{\partial d_{i}}
$$

The second derivatives of the structural mass can be obtained by further differentiating Eq. (54) with respect to the design variables, 


$$
\frac{\partial^{2} w(\mathbf{d})}{\partial d_{i} \partial d_{j}}=\sum_{e=1}^{N} \rho l_{e} \frac{\partial^{2} A^{e}}{\partial d_{i} \partial d_{j}}
$$

\section{Optimization mathematical model}

In general, we aim to minimize the total mass $w(\mathbf{d})$ of the structure. At the same time, the normal stresses, shear stresses, and nodal displacements should satisfy the constraints in the duration of transient loads. We formulate the optimization problem of the structures subjected to transient loads as follows.

$$
\begin{aligned}
& \text { Find } \mathbf{d} \\
& \min w(\mathbf{d}) \\
& \text { s.t. }-[\sigma] \leqslant \sigma_{i x}^{e+}(\mathbf{d}, t) \leqslant[\sigma] \quad(e=1,2, \cdots, N, t \in[0, \Gamma]) \\
& -[\sigma] \leqslant \sigma_{i x}^{e-}(\mathbf{d}, t) \leqslant[\sigma] \quad(e=1,2, \cdots, N, t \in[0, \Gamma]) \\
& -[\sigma] \leqslant \sigma_{j x}^{e+}(\mathbf{d}, t) \leqslant[\sigma] \quad(e=1,2, \cdots, N, t \in[0, \Gamma]) \\
& -[\sigma] \leqslant \sigma_{j x}^{e-}(\mathbf{d}, t) \leqslant[\sigma] \quad(e=1,2, \cdots, N, t \in[0, \Gamma]) \\
& -[\sigma] \leqslant \sigma_{M x}^{e+}(\mathbf{d}, t) \leqslant[\sigma] \quad(e=1,2, \cdots, N, t \in[0, \Gamma]) \\
& -[\sigma] \leqslant \sigma_{M x}^{e-}(\mathbf{d}, t) \leqslant[\sigma] \quad(e=1,2, \cdots, N, t \in[0, \Gamma]) \\
& -[\tau] \leqslant \tau_{i x y}^{e}(\mathbf{d}, t) \leqslant[\tau] \quad(e=1,2, \cdots, N, t \in[0, \Gamma]) \\
& -[\tau] \leqslant \tau_{j x y}^{e}(\mathbf{d}, t) \leqslant[\tau] \quad(e=1,2, \cdots, N, t \in[0, \Gamma]) \\
& -[\tau] \leqslant \tau_{M x y}^{e}(\mathbf{d}, t) \leqslant[\tau] \quad(e=1,2, \cdots, N, t \in[0, \Gamma]) \\
& -\left[x_{k}\right] \leqslant x_{k}(\mathbf{d}, t) \leqslant\left[x_{k}\right] \quad\left(k=1,2, \cdots, N_{f}\right) \\
& d_{J} \leqslant d_{J} \leqslant \bar{d}_{J}(J=1,2, \cdots, 4 N)
\end{aligned}
$$

where ' + ' and '-' are tension and compression, respectively. $[\sigma]$ is allowable normal stress. $[\tau]$ is allowable shear stress. $\Gamma$ is the duration of transient loads. $N_{f}$ is the number of degree of freedom. $\left[x_{k}\right]$ is allowable displacement on the $k$ th degree of freedom. $d_{J}$ is the lower limit of the $J$ th design variable. $\bar{d}_{J}$ is the upper limit of the $J$ th design variable.

Normalizing the constraints of the optimal model Eq. (56), we obtain the new equivalent mathematic model as follows.

Find d

$\min w(\mathbf{d})$

s.t. $g_{e}(\mathbf{d}, t)=\frac{\sigma_{i x}^{e+}(\mathbf{d}, t)}{[\sigma]}-1 \leqslant 0(e=1,2, \cdots, N ; t \in[0, \Gamma])$

$g_{e+N}(\mathbf{d}, t)=-\frac{\sigma_{i x}^{e+}(\mathbf{d}, t)}{[\sigma]}-1 \leqslant 0(e=1,2, \cdots, N ; t \in[0, \Gamma])$

$g_{e+2 N}(\mathbf{d}, t)=\frac{\sigma_{i x}^{e-}(\mathbf{d}, t)}{[\sigma]}-1 \leqslant 0(e=1,2, \cdots, N ; t \in[0, \Gamma])$

$g_{e+3 N}(\mathbf{d}, t)=-\frac{\sigma_{i x}^{e-}(\mathbf{d}, t)}{[\sigma]}-1 \leqslant 0(e=1,2, \cdots, N ; t \in[0, \Gamma])$

$g_{e+4 N}(\mathbf{d}, t)=\frac{\sigma_{j x}^{e+}(\mathbf{d}, t)}{[\sigma]}-1 \leqslant 0(e=1,2, \cdots, N ; t \in[0, \Gamma])$

$g_{e+5 N}(\mathbf{d}, t)=-\frac{\sigma_{j x}^{e+}(\mathbf{d}, t)}{[\sigma]}-1 \leqslant 0(e=1,2, \cdots, N ; t \in[0, \Gamma])$ 


$$
\begin{aligned}
& g_{e+6 N}(\mathbf{d}, t)=\frac{\sigma_{j x}^{e-}(\mathbf{d}, t)}{[\sigma]}-1 \leqslant 0(e=1,2, \cdots, N ; t \in[0, \Gamma]) \\
& g_{e+7 N}(\mathbf{d}, t)=-\frac{\sigma_{j x}^{e-}(\mathbf{d}, t)}{[\sigma]}-1 \leqslant 0(e=1,2, \cdots, N ; t \in[0, \Gamma]) \\
& g_{e+8 N}(\mathbf{d}, t)=\frac{\sigma_{M x}^{e+}(\mathbf{d}, t)}{[\sigma]}-1 \leqslant 0(e=1,2, \cdots, N ; t \in[0, \Gamma]) \\
& g_{e+9 N}(\mathbf{d}, t)=-\frac{\sigma_{M x}^{e+}(\mathbf{d}, t)}{[\sigma]}-1 \leqslant 0(e=1,2, \cdots, N ; t \in[0, \Gamma]) \\
& g_{e+10 N}(\mathbf{d}, t)=\frac{\sigma_{M x}^{e-}(\mathbf{d}, t)}{[\sigma]}-1 \leqslant 0(e=1,2, \cdots, N ; t \in[0, \Gamma]) \\
& g_{e+11 N}(\mathbf{d}, t)=-\frac{\sigma_{M x}^{e-}(\mathbf{d}, t)}{[\sigma]}-1 \leqslant 0(e=1,2, \cdots, N ; t \in[0, \Gamma]) \\
& g_{e+12 N}(\mathbf{d}, t)=\frac{\tau_{i x y}^{e}(\mathbf{d}, t)}{[\tau]}-1 \leqslant 0(e=1,2, \cdots, N ; t \in[0, \Gamma]) \\
& g_{e+13 N}(\mathbf{d}, t)=-\frac{\tau_{i x y}^{e}(\mathbf{d}, t)}{[\tau]}-1 \leqslant 0(e=1,2, \cdots, N ; t \in[0, \Gamma]) \\
& g_{e+14 N}(\mathbf{d}, t)=\frac{\tau_{j x y}^{e}(\mathbf{d}, t)}{[\tau]}-1 \leqslant 0(e=1,2, \cdots, N ; t \in[0, \Gamma]) \\
& g_{e+15 N}(\mathbf{d}, t)=-\frac{\tau_{j x y}^{e}(\mathbf{d}, t)}{[\tau]}-1 \leqslant 0(e=1,2, \cdots, N ; t \in[0, \Gamma]) \\
& g_{e+16 N}(\mathbf{d}, t)=\frac{\tau_{M x y}^{e}(\mathbf{d}, t)}{[\tau]}-1 \leqslant 0(e=1,2, \cdots, N ; t \in[0, \Gamma]) \\
& g_{e+17 N}(\mathbf{d}, t)=-\frac{\tau_{M x y}^{e}(\mathbf{d}, t)}{[\tau]}-1 \leqslant 0(e=1,2, \cdots, N ; t \in[0, \Gamma]) \\
& g_{k+18 N}(\mathbf{d}, t)=\frac{x_{k}(\mathbf{d}, t)}{\left[x_{k}\right]}-1 \leq 0\left(k=1,2, \cdots, N_{f}\right) \\
& g_{k+N_{f}+18 N}(\mathbf{d}, t)=-\frac{x_{k}(\mathbf{d}, t)}{\left[x_{k}\right]}-1 \leqslant 0\left(k=1,2, \cdots, N_{f}\right) \\
& g_{J}(\mathbf{d})=\frac{d_{J}}{d_{J}}-1 \leqslant 0(J=1,2, \cdots, 4 N) \\
& g_{J+4 N}(\mathbf{d})=1-\frac{d_{J}}{d_{J}} \leqslant 0(J=1,2, \cdots, 4 N)
\end{aligned}
$$

\section{Transformation of mathematical model}

The key feature of the transformation method is to transform a constrained problem into an unconstrained problem. Thus, we minimize only one function in the transformation method. This is an attractive aspect in that many time-dependent constraints and an objective function can be merged into a single function. The representatives of the transformation method are the augmented Lagrange multiplier method [16] and the exterior penalty function method [20] in structure optimal design under dynamic loads. However, the augmented Lagrange multiplier function and the exterior penalty function are discontinuous functions, therefore, the gradient and Hessian matrix calculations of these functions are difficult when the direct differentiation method is employed to obtain the first and second derivatives. Compared to the augmented Lagrange multiplier function and the exterior penalty function, the interior penalty function is a continuous function, so the gradient and Hessian matrix calculations of the interior penalty function are relatively easy when the direct differentiation method is used to obtain the first and second derivatives. The interior penalty function method requires a feasible initial design point. Typically, it may be difficult to obtain a feasible initial design in a complex problem. However, in structural optimization problems, a feasible design point can be found in the structures with the large cross-sectional areas. Therefore, in this paper the interior penalty function is employed to transform the inequality constraint optimization problem. 


\subsection{Interior penalty function method}

In this paper£<the inequality constraint optimal mathematic model Eq. (57) is converted to a sequence of appropriately formed unconstrained integral mathematic model using the interior penalty function method. The interior penalty function method is adopted as follows.

$$
P\left(\mathbf{d}, r_{k}\right)=w(\mathbf{d})-r_{k}\left(\sum_{J=1}^{2 N_{f}+18 N} \int_{0}^{\Gamma} \frac{1}{g_{J}(\mathbf{d}, t)} d t+\sum_{I=1}^{8 N} \frac{1}{g_{I}(\mathbf{d})}\right)
$$

In Eq. (58), penalty parameter $r_{k}$ is a sequence of numbers which are degressive. When $r_{k} \rightarrow 0$, the minimum of penalty function $P\left(\mathbf{d}, r_{k}\right)$ approaches the minimum of the constraint problem. So the solution of the inequality constraint optimization model, Eq. (57), is transformed into a sequence of unconstraint problems:

Find $\mathbf{d}$

$$
\min P\left(\mathbf{d}, r_{k}\right)=w(\mathbf{d})-r_{k}\left(\sum_{J=1}^{2 N_{f}+18 N} \int_{0}^{\Gamma} \frac{1}{g_{J}(\mathbf{d}, t)} d t+\sum_{I=1}^{8 N} \frac{1}{g_{I}(\mathbf{d})}\right)
$$

Initial penalty parameter $r_{1}$ can be calculated by the following equation,

$$
r_{1}\left(\sum_{J=1}^{2 N_{f}+18 N} \int_{0}^{\Gamma} \frac{1}{g_{J}\left(\mathbf{d}_{0}, t\right)} d t+\sum_{I=1}^{8 N} \frac{1}{g_{I}\left(\mathbf{d}_{0}\right)}\right)=\frac{p_{0}}{100} w\left(\mathbf{d}_{0}\right)
$$

where $\mathbf{d}_{0}$ is the initial design point and $p_{0}=1 \sim 50$. In this work, we choose $p_{0}=50$.

The penalty parameter $r_{k}$ decreases according to the following rule:

$$
r_{k+1}=\frac{r_{k}}{c}
$$

where $c=10 \sim 50$ and $c=10$ in this work. $k$ is the number of penalty parameter which will be used in the process of search.

\subsection{Calculation of gradient and Hessian matrix of interior penalty function}

Now we calculate the first and second derivatives of the penalty function with respect to the structural design variables. The time step and duration of dynamic loads are $\Delta t$ and $\Gamma$, respectively. Let $a=\frac{\Gamma}{\Delta t}$.

The first derivatives of penalty function can be obtained by differentiating Eq. (58) with respect to the design variable $d_{i}$,

$$
\frac{\partial P\left(\mathbf{d}, r_{k}\right)}{\partial d_{i}}=\frac{\partial w(\mathbf{d})}{\partial d_{i}}+r_{k}\left(\sum_{J=1}^{2 N_{f}+18 N} \int_{0}^{\Gamma} \frac{1}{g_{J}^{2}(\mathbf{d}, t)} \frac{\partial g_{J}(\mathbf{d}, t)}{\partial d_{i}} d t+\sum_{I=1}^{8 N} \frac{1}{g_{I}^{2}(\mathbf{d})} \frac{\partial g_{I}(\mathbf{d})}{\partial d_{i}}\right)
$$

The second derivatives of penalty function is calculated by further differentiating Eq. (62) with respect to the design variable $d_{j}$,

$$
\begin{aligned}
& \frac{\partial^{2} P\left(\mathbf{d}, r_{k}\right)}{\partial d_{i} \partial d_{j}}=\frac{\partial^{2} w(\mathbf{d})}{\partial d_{i} \partial d_{j}}+r_{k} \sum_{J=1}^{2 N_{f}+18 N} \int_{0}^{\Gamma}\left[\frac{-2}{g_{J}^{3}(\mathbf{d}, t)} \frac{\partial g_{J}(\mathbf{d}, t)}{\partial d_{j}} \frac{\partial g_{J}(\mathbf{d}, t)}{\partial d_{i}}+\frac{1}{g_{J}^{2}(\mathbf{d}, t)} \frac{\partial^{2} g_{J}(\mathbf{d}, t)}{\partial d_{i} \partial d_{j}}\right] d t \\
& +r_{k} \sum_{I=1}^{8 N} \frac{-2}{g_{I}^{3}(\mathbf{d})} \frac{\partial g_{I}(\mathbf{d})}{\partial d_{j}} \frac{\partial g_{I}(\mathbf{d})}{\partial d_{i}}
\end{aligned}
$$

The integral terms in Eqs (58), (59), (60), (62), (63) are computed by using the trapezoidal form integral formula:

$$
\int_{0}^{\Gamma} \frac{1}{g_{J}(\mathbf{d}, t)} d t=\sum_{z=0}^{a-1} \frac{\Delta t}{2}\left[\frac{1}{g_{J}(\mathbf{d}, z \Delta t)}+\frac{1}{g_{J}(\mathbf{d},(z+1) \Delta t)}\right]
$$




$$
\begin{aligned}
\int_{0}^{\Gamma} & \frac{1}{g_{J}^{2}(\mathbf{d}, t)} \frac{\partial g_{J}(\mathbf{d}, t)}{\partial d_{i}} d t=\sum_{z=0}^{a-1} \frac{\Delta t}{2}\left[\frac{1}{g_{J}^{2}(\mathbf{d}, z \Delta t)} \frac{\partial g_{J}(\mathbf{d}, z \Delta t)}{\partial d_{i}}+\frac{1}{g_{J}^{2}(\mathbf{d},(z+1) \Delta t)} \frac{\partial g_{J}(\mathbf{d},(z+1) \Delta t)}{\partial d_{i}}\right] \\
& \int_{0}^{\Gamma}\left[\frac{-2}{g_{J}^{3}(\mathbf{d}, t)} \frac{\partial g_{J}(\mathbf{d}, t)}{\partial d_{j}} \frac{\partial g_{J}(\mathbf{d}, t)}{\partial d_{i}}+\frac{1}{g_{J}^{2}(\mathbf{d}, t)} \frac{\partial^{2} g_{J}(\mathbf{d}, t)}{\partial d_{i} \partial d_{j}}\right] d t= \\
& \sum_{z=0}^{a-1} \frac{\Delta t}{2}\left\{\left[\frac{-2}{g_{J}^{3}(\mathbf{d}, z \Delta t)} \frac{\partial g_{J}(\mathbf{d}, z \Delta t)}{\partial d_{j}} \frac{\partial g_{J}(\mathbf{d}, z \Delta t)}{\partial d_{i}}+\frac{1}{g_{c}^{2}(\mathbf{d}, z \Delta t)} \frac{\partial^{2} g_{J}(\mathbf{d}, z \Delta t)}{\partial d_{i} \partial d_{j}}\right]+\right. \\
& {\left.\left[\frac{-2}{g_{J}^{3}(\mathbf{d},(z+1) \Delta t)} \frac{\partial g_{J}(\mathbf{d},(z+1) \Delta t)}{\partial d_{j}} \frac{\partial g_{J}(\mathbf{d},(z+1) \Delta t)}{\partial d_{i}}+\frac{1}{g_{J}^{2}(\mathbf{d},(z+1) \Delta t)} \frac{\partial^{2} g_{J}(\mathbf{d},(z+1) \Delta t)}{\partial d_{i} \partial d_{j}}\right]\right\} }
\end{aligned}
$$

The first and second derivatives of the penalty function with respect to the structural variables are calculated. Then the gradient and Hessian matrix can be achieved.

\section{Solving optimization problems}

The inequality constraint optimization model, Eq. (57), is converted into a sequence of the appropriately formed unconstrained integral model, Eq. (59). Marquardt's method, a gradient-Hessian matrix-based algorithm, is adopted to solve the unconstrained problem, taking advantages that the gradient and Hessian matrixes are fully used in this optimal method. Marquardt's method combines Cauchy's and Newton's methods in a convenient manner that exploits the strengths of both but does require second-order information. The major merit of Marquardt's method is its simplicity, descent property, excellent convergence rate near the optimum, and absence of a line search. Based on Marquardt's method, the computation procedure of solving the mathematic model Eq. (56) is as follows.

The computer procedure of solving the mathematic model Eq. (56):

Step 1. Chose the initial feasible design point $\mathbf{d}_{0}$, calculate $r_{1}$ by solving Eq. (60), define convergence criterion $\varepsilon_{1}$, let $k=1$.

Step 2. Start from design point $\mathbf{d}_{k-1}$, solve the mathematic model Eq. (59) with Marquardt's method to obtain the optimum design $\mathbf{d}_{k}$.The steps of solving the mathematic model Eq. (59) with Marquardt's method is from Step 2.1. to Step 2.11.:

Step 2.1. Let $\mathbf{d}_{k-1}^{(0)}=\mathbf{d}_{k-1}$. Define

$M_{I}=$ maximum number of iterations allowed

$\varepsilon_{2}=$ convergence criterion

$\mathbf{I}=$ identity matrix

Step 2.2. Set $i=0 . \lambda^{(0)}=10^{5}$.

Step 2.3. Calculate $\nabla P\left(\mathbf{d}_{k-1}^{(i)}, r_{k}\right)$.

Step 2.4. Is $\left\|\nabla P\left(\mathbf{d}_{k-1}^{(i)}, r_{k}\right)\right\| \leqslant \varepsilon_{2}$ ?

Yes: Go to step 2.11.

No: Continue.

Step 2.5. Is $i \geqslant M_{I}$ ?

Yes: Go to step 2.11.

No: Continue.

Step 2.6. Calculate $\mathbf{S}\left(\mathbf{d}_{k-1}^{(i)}\right)=-\left[\nabla^{2} P\left(\mathbf{d}_{k-1}^{(i)}, r_{k}\right)+\lambda^{(i)} \mathbf{I}\right]^{-1} \nabla P\left(\mathbf{d}_{k-1}^{(i)}, r_{k}\right)$.

Step 2.7. Set $\mathbf{d}_{k-1}^{(i+1)}=\mathbf{d}_{k-1}^{(i)}+\mathbf{S}\left(\mathbf{d}_{k-1}^{(i)}\right)$.

Step 2.8. Is $P\left(\mathbf{d}_{k-1}^{(i+1)}, r_{k}\right)<P\left(\mathbf{d}_{k-1}^{(i)}, r_{k}\right)$ ?

Yes: Go to step 2.9. 
No: Go to step 2.10.

Step 2.9. Set $\lambda^{(i+1)}=\frac{1}{2} \lambda^{(i)}$ and $i=i+1$. Go to step 2.3.

Step 2.10. Set $\lambda^{(i)}=2 \lambda^{(i)}$. Go to step 2.6.

Step 2.11. Set $\mathbf{d}_{k}=\mathbf{d}_{k-1}^{(i)}$. Go to step 3 .

Step 3. Is $P\left(\mathbf{d}_{k-1}, r_{k}\right)-P\left(\mathbf{d}_{k}, r_{k}\right) \leqslant \varepsilon_{1}$ ?

Yes: $\mathbf{d}_{k}$ is the best design. Print result and stop.

No: Continue.

Step 4. Calculate $r_{k+1}$ by solving Eq. (61), $k=k+1$. Go to step 2 .

\section{Numerical example}

In this section, optimization design of the plane frame shown in Fig. 2 is performed using the method proposed in this paper. The plane frame is divided with three plane beam elements. The cross-section of the element is $\mathrm{H}$ structural-steel shape, shown in Fig. 1. We use typical steel materials properties, i.e., elastic modulus $E=210 \mathrm{GPa}$ and material density $\rho=7850 \mathrm{~kg} / \mathrm{m}^{3}$, for all elements. The structural damping ratios are $\varsigma_{1}=0.02, \varsigma_{2}=0.02$. The horizontal dynamic load, $F_{h}(t)=1500 \sin \frac{3 \pi}{4} t(\mathrm{kN})$, acts on the node 2 . A duration of $3 \mathrm{~s}$ and an incremental time step of $0.01 \mathrm{~s}$ are considered in optimization procedures. The convergence criterion: $\varepsilon_{1}=10^{-3}, \varepsilon_{2}=10^{-3}$ and the maximum number of iterations allowed: $M_{I}=5$. Numerical tests show that these criteria are sufficient for achieving convergence in a reasonable time.

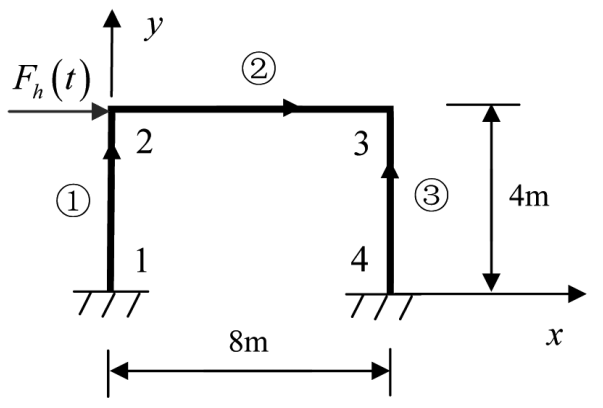

Fig. 2. Plane frame.

In the optimal mathematic model Eq. (57), the allowable normal stress $[\sigma]=200 \mathrm{MPa}$ and allowable shear stress $[\tau]=100 \mathrm{MPa}$; the nodal allowable displacements are: $\left[x_{1}\right]=0.001 \mathrm{~m},\left[x_{2}\right]=0.001 \mathrm{~m},\left[x_{3}\right]=0.1 \mathrm{rad}$, $\left[x_{4}\right]=0.001 \mathrm{~m},\left[x_{5}\right]=0.001 \mathrm{~m},\left[x_{6}\right]=0.1 \mathrm{rad}$; the design variable vector is defined as: $\mathbf{d}=\left[d_{1}^{1}, d_{2}^{1}\right.$, $\left.d_{3}^{1}, d_{4}^{1}, d_{1}^{2}, d_{2}^{2}, d_{3}^{2}, d_{4}^{2}, d_{1}^{3}, d_{2}^{3}, d_{3}^{3}, d_{4}^{3}\right]^{T}$. The design space is shown in Table 1 .

Table 1

Design space of frame (unit: $\mathrm{mm}$ )

\begin{tabular}{lcccccccccccc}
\hline Design variables & $d_{1}^{1}$ & $d_{2}^{1}$ & $d_{3}^{1}$ & $d_{4}^{1}$ & $d_{1}^{2}$ & $d_{2}^{2}$ & $d_{3}^{2}$ & $d_{4}^{2}$ & $d_{1}^{3}$ & $d_{2}^{3}$ & $d_{3}^{3}$ & $d_{4}^{3}$ \\
\hline Lower limit & 100 & 100 & 4 & 4 & 100 & 100 & 4 & 4 & 100 & 100 & 4 & 4 \\
Upper limit & 500 & 1000 & 70 & 70 & 500 & 1000 & 70 & 70 & 500 & 1000 & 70 & 70 \\
\hline
\end{tabular}

The optimum designs of the plane frame are searched from two different initial feasible design points. Sequences of optimum designs are shown in Tables 2 and 3.

From the optimum results shown in Tables 2 and 3, the masses of the optimum designs approach to the final targets, $26 \mathrm{~kg}$ and $16 \mathrm{~kg}$, from the initial feasible design 1 (i.e., $153 \mathrm{~kg}$ ) and 2 (i.e., $45 \mathrm{~kg}$ ), respectively. It indicates that the optimization method presented in this paper is effective. However, the optimum designs are different if the initial designs are not same. Therefore, the optimum designs obtained with the optimization method in this paper are local solutions, but not global solutions. We should find the different local solutions from as many initial designs as possible. Then we can choose the best design from the local designs as the effective design. 
Table 2

Initial feasible design 1 and optimum design (unit: $\mathrm{mm}$ )

\begin{tabular}{lccccccccccccccc}
\hline Design variables & & $d_{1}^{1}$ & $d_{2}^{1}$ & $d_{3}^{1}$ & $d_{4}^{1}$ & $d_{1}^{2}$ & $d_{2}^{2}$ & $d_{3}^{2}$ & $d_{4}^{2}$ & $d_{1}^{3}$ & $d_{2}^{3}$ & $d_{3}^{3}$ & $d_{4}^{3}$ & Mass/kg & Time/s \\
\hline Initial design 1 & & 450 & 900 & 60 & 60 & 450 & 900 & 60 & 60 & 450 & 900 & 60 & 60 & 153 & 0 \\
Method in this paper & $r_{1}=251.14$ & 342 & 801 & 45 & 46 & 263 & 785 & 42 & 39 & 294 & 794 & 45 & 44 & 63 & 2392 \\
& $r_{2}=25.114$ & 341 & 800 & 33 & 34 & 261 & 785 & 23 & 11 & 292 & 793 & 34 & 29 & 26 & 4770 \\
& $r_{3}=2.5114$ & 341 & 800 & 33 & 34 & 261 & 785 & 23 & 11 & 292 & 793 & 34 & 29 & 26 & 7149 \\
Augmented Lagrange multiplier & 363 & 820 & 29 & 32 & 276 & 779 & 18 & 10 & 300 & 810 & 38 & 27 & 24 & 8426 \\
\hline
\end{tabular}

Table 3

Initial feasible design 2 and optimum design (unit: $\mathrm{mm}$ )

\begin{tabular}{lccccccccccccccc}
\hline Design variables & & $d_{1}^{1}$ & $d_{2}^{1}$ & $d_{3}^{1}$ & $d_{4}^{1}$ & $d_{1}^{2}$ & $d_{2}^{2}$ & $d_{3}^{2}$ & $d_{4}^{2}$ & $d_{1}^{3}$ & $d_{2}^{3}$ & $d_{3}^{3}$ & $d_{4}^{3}$ & Mass/kg & Time/s \\
\hline Initial design 2 & & 300 & 600 & 40 & 40 & 300 & 600 & 40 & 40 & 300 & 600 & 40 & 40 & 45 & 0 \\
Method in this paper & $r_{1}=96.33$ & 303 & 600 & 30 & 37 & 298 & 599 & 18 & 18 & 298 & 599 & 27 & 27 & 24 & 2118 \\
& $r_{2}=9.633$ & 302 & 600 & 27 & 35 & 298 & 599 & 18 & 18 & 298 & 599 & 27 & 27 & 22 & 4235 \\
& $r_{3}=0.9633$ & 302 & 600 & 25 & 33 & 298 & 599 & 8 & 8 & 298 & 599 & 22 & 22 & 16 & 6558 \\
& $r_{4}=0.09633$ & 302 & 600 & 25 & 33 & 298 & 599 & 8 & 8 & 298 & 599 & 22 & 22 & 16 & 8896 \\
Augmented Lagrange multiplier & 300 & 596 & 26 & 31 & 298 & 598 & 8 & 7 & 294 & 593 & 25 & 27 & 16 & 11042 \\
\hline
\end{tabular}

All simulations are performed on a personal computer with Window XP operating system. The computer has a Pentium(R) 4 CPU, and frequency of the CPU is $2.8 \mathrm{GHz}$. The computer also has 512MB RAM. The iteration courses shown in Fig. 3 indicate that the algorithm is convergent. From initial feasible design 1, the computational time of achieving the local optimum design is about $7149 \mathrm{~s}$ with the method in this paper, and about $8426 \mathrm{~s}$ with the Augmented Lagrange multiplier method. From initial feasible design 2, the computational time of achieving the local optimum design is about $8896 \mathrm{~s}$ with the method in this paper, and about $11042 \mathrm{~s}$ with the Augmented Lagrange multiplier method. The computational time shows that the method proposed in this paper is sometimes more efficient than the Augmented Lagrange multiplier method.

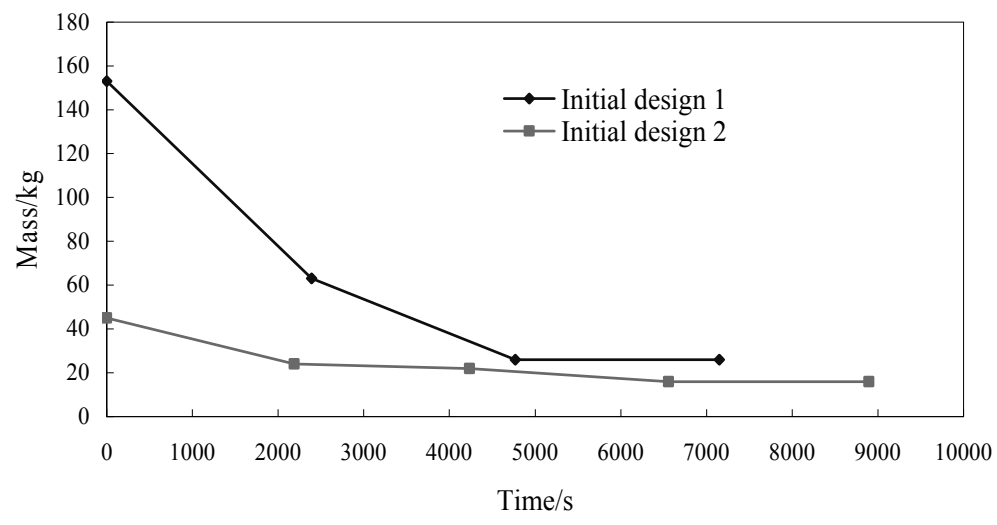

Fig. 3. Iteration courses.

It should be noted that the gradient and Hessian matrix calculation is very difficult for the dynamic optimization problem because it is difficult to calculate the dynamic response first and second derivatives. Generally, the gradient and Hessian matrix calculation requires much computational time that can not be accepted in the structural optimization. However, in this work we develop an algorithm, only a single dynamics analysis is required, to obtain the gradient and Hessian matrix. In addition, we use the interior penalty function method to transform a constrained problem into an unconstrained problem. So many time-dependent constraints and an objective function can be merged into a sequence of appropriately formed unconstrained integral single time-independent functions. Those unconstrained integral single time-independent functions are continuous, so the gradient and Hessian matrix calculations are easier than other discontinuous transform functions, the augmented Lagrange multiplier function and exterior penalty function. 


\section{Conclusions}

In this paper, we developed an optimization method of structures subjected to transient loads. The conclusions are as follows.

(1) An algorithm is formulated to calculate structural dynamic responses, i.e., nodal displacements and stresses, and their first and second derivatives with respect to design variables. The algorithm is achieved by direct differentiation and only a single dynamics analysis based on Newmark- $\beta$ method is required.

(2) The interior penalty function method is used to transform a constrained problem into an unconstrained problem. So many time-dependent constraints and an objective function can be merged into a sequence of appropriately formed unconstrained integral single time-independent functions. Those unconstrained integral single time-independent functions are continuous, so the gradient and Hessian matrix calculations are easier than other discontinuous transform functions, the augmented Lagrange multiplier function and exterior penalty function.

(3) The gradient-Hessian matrix-based optimization method presented in this paper has the characteristics of its simplicity, descent property, excellent convergence rate near the optimum, and absence of a line search.

(4) The numerical results show that the optimum designs obtained with the optimization method presented in this paper are local solutions, but not global solutions and that the optimization method is effective.

(5) The numerical results also show that the optimal design method proposed in this paper is sometimes more efficient than the augmented Lagrange multiplier method. Sometimes, it depends on the chosen initial design.

\section{References}

[1] F.Y. Kocer and J.S. Arora, Optimal design of h-frame transmission poles for earthquake loading, Journal of Structure Engineering 125 (1999), 1299-1308.

[2] A.E. Baumal, J.J. McPhee and P.H. Calamai, Application of genetic algorithms to the design optimization of an active vehicle suspension system, Computer Methods in Applied Mechanics and Engineering 163 (1998), 87-94.

[3] C.P. Pantelides and S.R. Tsan, Optimal design of dynamically constraints structures, Computers and Structures 62 (1997), 141-150.

[4] I. Bucher, Parametric optimization of structures under combined base motion direct forces and static loading, Journal of Vibration and Acoustics Transactions of the ASME 124 (2002), 132-140.

[5] F. van Keulen, R.T. Haftka and N.H. Kim, Review of options for structural design sensitivity analysis. Part 1: Linear systems, Computer Methods in Applied Mechanics and Engineering 194 (2005), 3213-3243.

[6] C.C. Hsieh and J.S. Arora, Design sensitivity analysis and optimization of dynamic response, Computer Methods in Applied Mechanics and Engineering 43 (1984), 195-219.

[7] J.L. Chen and J.S. Ho, Direct variational method for sizing design sensitivity analysis of beam and frame structures, Computers and Structures 42 (1992), 503-509.

[8] K. Kulkarni and A.K. Noor, Sensitivity analysis for the dynamic response of viscoplastic shells of revolution, Computers and Structures 55 (1995), 955-969.

[9] M. Bogomolni, U. Kirsch and I. Sheinman, Efficient design sensitivities of structures subjected to dynamic loading, International Journal of Solids and Structures 43 (2006), 5485-5500.

[10] U. Kirsch, M. Bogomolni and F. van Keulen, Efficient finite-difference design-sensitivities, AIAA Journal 43 (2005), $399-405$.

[11] U. Kirsch and P.Y. Papalambros, Accurate displacement derivatives for structural optimization using approximate reanalysis, Computer Methods in Applied Mechanics and Engineering 190 (2001), 3945-3956.

[12] K.W. Lee and G.J. Park, Accuracy test of sensitivity analysis in the semi-analytic method with respect to configuration variables, Computers and Structures 63 (1997), 1139-4148.

[13] B.S. Kang, G.J. Park and J.S. Arora, A review of optimization of structures subjected to transient loads, Structural and Multidisciplinary Optimization 31 (2006), 81-95.

[14] J.S. Arora and J.E.B. Cardoso, A design sensitivity analysis principle and its implementation into ADINA, Computers and Structures 32 (1989), 691-705.

[15] C.C. Hsieh and J.S. Arora, A hybrid formulation for treatment of point-wise state variable constraints in dynamic response optimization, Computer Methods in Applied Mechanics and Engineering 48 (1985), 171-189.

[16] A.I. Chahande and J.S. Arora, Development of a multiplier method for dynamic response optimization problem, Structural Optimization 6 (1993), 69-78.

[17] C.P. Pantelides and S.R. Tsan, Optimal design of dynamically constraints structures, Computers and Structures 62 (1997), 141-150.

[18] N.M. Newmark, A Method of Computation for structural dynamics, Journal of Engineer Mechanics Division 85 (1959), 67-94.

[19] G.V. Reklaitis, A. Ravindran and K.M. Ragsdell, Engineering Optimization Methods and Applications, John Wiley and Sons, New York, 1983.

[20] J.H. Cassis and L.A. Schmit, Optimum structural design with dynamic constraints, Journal of Structural Engineering Proceedings ASCE 102 (1976), 2053-2071. 

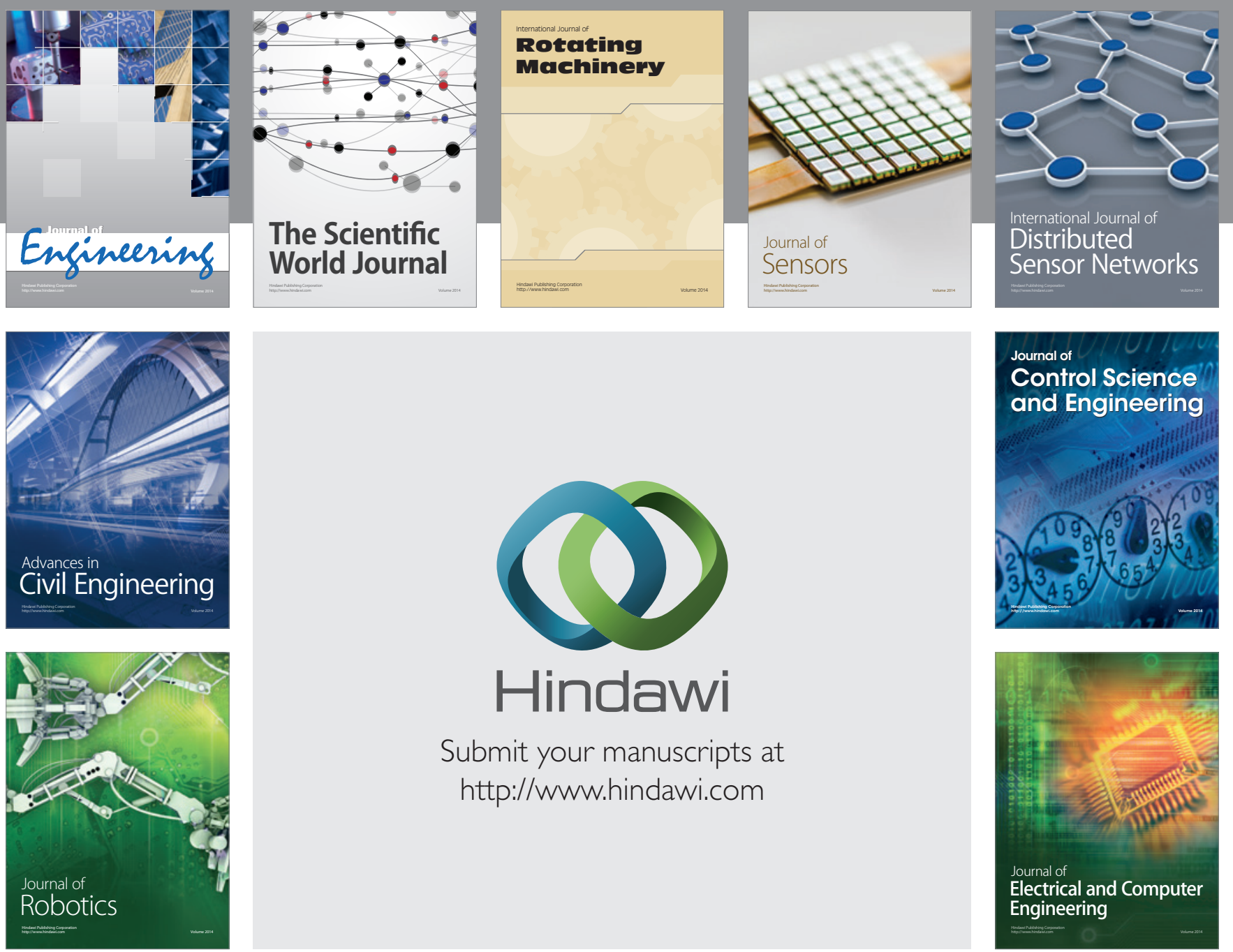

Submit your manuscripts at

http://www.hindawi.com
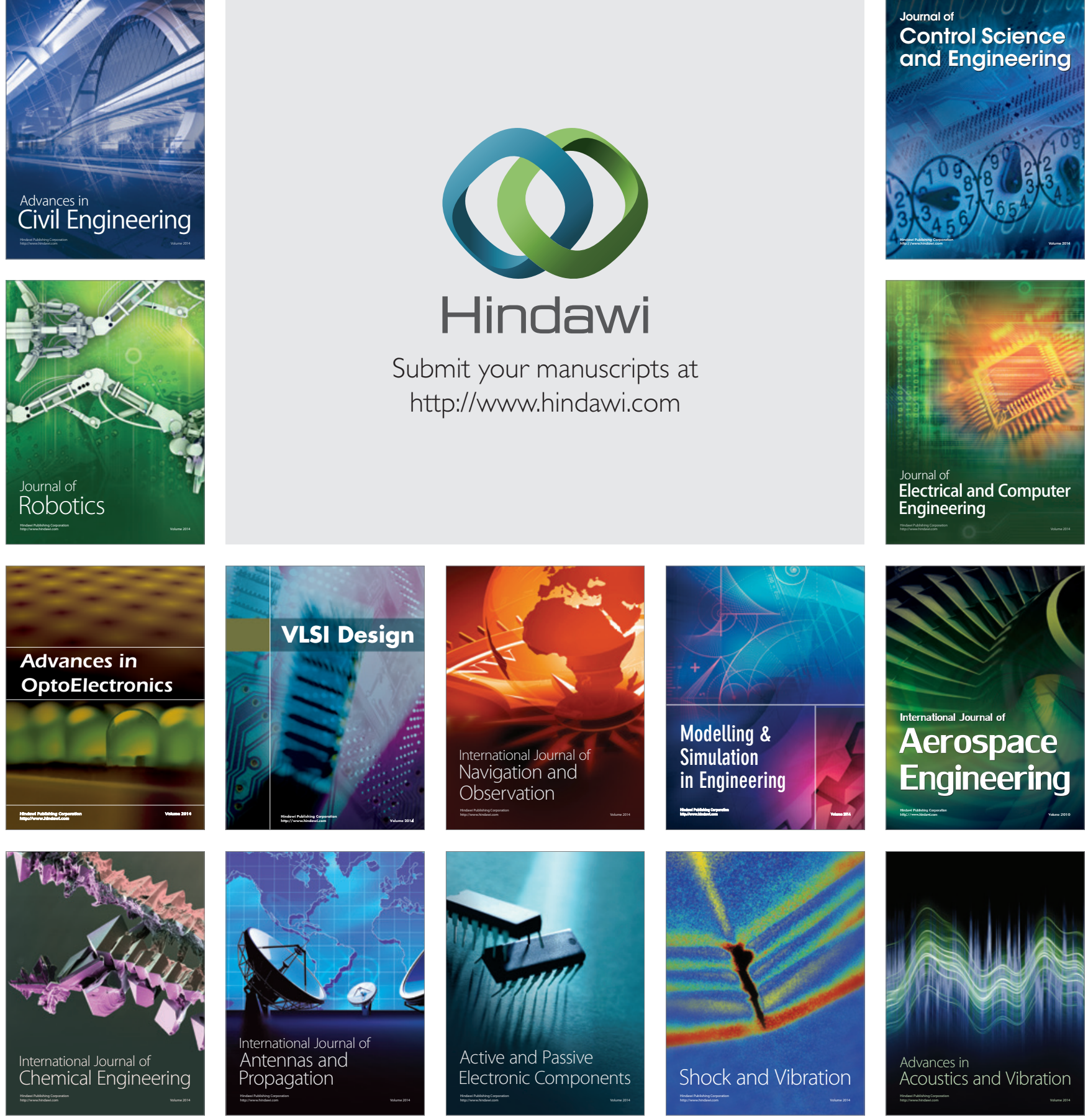